Acta Crystallographica Section E

Structure Reports

Online

ISSN 1600-5368

\section{Bis[N-cyclohexyl-1-(2-\{1-[(cyclohexyl- amino)carbonyl]cyclohexyl\}-3,5-dioxo-} 1,2-oxazolidin-4-yl)cyclopentanecarboxamide] monohydrate

\section{Enayatollah Sheikhhosseini, ${ }^{a}$ Fahime Vafadarnejad ${ }^{b}$ and Azizollah Habibi ${ }^{\text {b* }}$}

${ }^{a}$ Faculty of Science, Department of Chemistry, University of Islamic Azad, Kerman Branch, Kerman, Iran, and ${ }^{\mathbf{b}}$ Faculty of Chemistry, Tarbiat Moallem University, 49 Mofateh Avenue, Tehran, Iran

Correspondence e-mail: habibi@tmu.ac.ir

Received 18 May 2011; accepted 18 July 2011

Key indicators: single-crystal X-ray study; $T=100 \mathrm{~K}$; mean $\sigma(\mathrm{C}-\mathrm{C})=0.003 \AA$; disorder in main residue; $R$ factor $=0.047 ; w R$ factor $=0.120$; data-to-parameter ratio $=17.7$.

The reaction of cyclohexyl isocyanide and alkylidene Meldrum's acid (systematic name 2,2-dimethyl-1,3-dioxane4,6-dione) in the presence of cyclohexyl ketoxime and dichloromethane as solvent resulted in the title compound, $2 \mathrm{C}_{28} \mathrm{H}_{43} \mathrm{~N}_{3} \mathrm{O}_{5} \cdot \mathrm{H}_{2} \mathrm{O}$. One methylene group of the cyclopentane ring was found to be disordered and was refined with occupancies 0.75:0.25. Intramolecular $\mathrm{N}-\mathrm{H} \cdots \mathrm{O}$ hydrogen bonds occur. The crystal structure is stabilized by intermolecular $\mathrm{N}-\mathrm{H} \cdots \mathrm{O}$ and $\mathrm{O}-\mathrm{H} \cdots \mathrm{O}$ hydrogen bonds.

\section{Related literature}

For the biological activity of isoxazoles, see: Conti et al. (1998); Kang et al. (2000); Ko et al. (1998); Mishra et al. (1998).

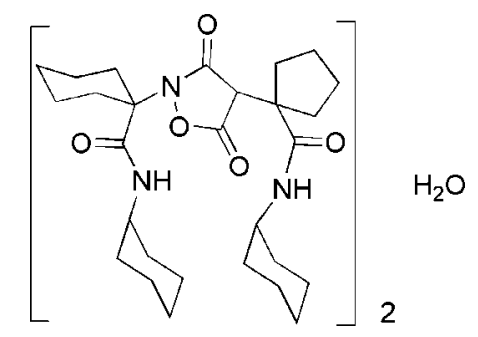

\section{Experimental}

Crystal data

$\begin{array}{ll}2 \mathrm{C}_{28} \mathrm{H}_{43} \mathrm{~N}_{3} \mathrm{O}_{5} \cdot \mathrm{H}_{2} \mathrm{O} & \gamma=93.100(1)^{\circ} \\ M_{r}=1021.32 & V=2733.9(3) \AA^{3} \\ \text { Triclinic, } P \overline{1} & Z=2 \\ a=11.3084(6) \AA & \text { Mo } K \alpha \text { radiation } \\ b=12.3119(7) \AA & \mu=0.09 \mathrm{~mm}^{-1} \\ c=20.7904(11) \AA & T=100 \mathrm{~K} \\ \alpha=102.686(1)^{\circ} & 0.60 \times 0.50 \times 0.10 \mathrm{~mm}\end{array}$

$\beta=103.104(1)^{\circ}$

Data collection

Bruker APEXII CCD

diffractometer

28848 measured reflections

11931 independent reflections 9099 reflections with $I>2 \sigma(I)$ $R_{\text {int }}=0.028$

Refinement

$R\left[F^{2}>2 \sigma\left(F^{2}\right)\right]=0.047$

$w R\left(F^{2}\right)=0.120$

$S=1.03$

11931 reflections

675 parameters
$\mathrm{H}$ atoms treated by a mixture of independent and constrained refinement
$\Delta \rho_{\max }=0.38$ e $\AA^{-3}$
$\Delta \rho_{\min }=-0.25 \mathrm{e}^{-3}$

Table 1

Hydrogen-bond geometry $\left(\AA{ }^{\circ}\right)$.

\begin{tabular}{lllll}
\hline$D-\mathrm{H} \cdots A$ & $D-\mathrm{H}$ & $\mathrm{H} \cdots A$ & $D \cdots A$ & $D-\mathrm{H} \cdots A$ \\
\hline $\mathrm{N} 2 A-\mathrm{H} 2 A A \cdots \mathrm{O} 5 A$ & 0.88 & 2.09 & $2.9371(18)$ & 161 \\
$\mathrm{~N} 3 A-\mathrm{H} 3 A A \cdots \mathrm{O} 1 W$ & 0.88 & 2.00 & $2.8662(19)$ & 170 \\
$\mathrm{~N} 2 B-\mathrm{H} 2 B A \cdots \mathrm{O} 5 B$ & 0.88 & 2.10 & $2.9330(18)$ & 157 \\
$\mathrm{~N} 3 B-\mathrm{H} 3 B A \cdots \mathrm{O} 3 A$ & 0.88 & 2.07 & $2.9238(17)$ & 163 \\
$\mathrm{O} 1 W-\mathrm{H} 1 W A \cdots \mathrm{O} 4 B^{\mathrm{i}}$ & $0.85(3)$ & $1.90(3)$ & $2.7433(18)$ & $169(2)$ \\
$\mathrm{O} 1 W-\mathrm{H} 1 W B \cdots \mathrm{O} 4 A^{\mathrm{ii}}$ & $0.89(2)$ & $1.88(2)$ & $2.7569(18)$ & $170(2)$ \\
\hline
\end{tabular}

Symmetry codes: (i) $-x+1,-y+2,-z+1$; (ii) $x+1, y, z$.

Data collection: APEX2 (Bruker, 2005); cell refinement: SAINT (Bruker, 2005); data reduction: $S A I N T$; $\operatorname{program}(\mathrm{s})$ used to solve structure: SHELXS97 (Sheldrick, 2008); program(s) used to refine structure: SHELXL97 (Sheldrick, 2008); molecular graphics: SHELXTL (Sheldrick, 2008); software used to prepare material for publication: SHELXTL.

Supplementary data and figures for this paper are available from the IUCr electronic archives (Reference: VM2097).

\title{
References
}

Bruker (2005). APEX2 and SAINT. Bruker AXS Inc., Madison, Wisconsin, USA.

Conti, P., Dallanoce, C., Amici, M. D., Micheli, C. D. \& Klotz, K.-N. (1998). Bioorg. Med. Chem. Lett. 6, 401-408.

Kang, Y. Y., Shin, K. J., Yoo, K. H., Seo, K. J., Hong, C. Y., Lee, C.-S., Park, S. Y., Kim, D. J. \& Park, S. W. (2000). Bioorg. Med. Chem. Lett. 10, 95-99. Ko, D.-H., Maponya, M. F., Khalil, M. A., Oriaku, E. T., You, Z. \& Lee, J. (1998). Med. Chem. Res. 8, 313-318.

Mishra, A., Jain, S. J. \& Asthana, J. G. (1998). Orient. J. Chem. 14, 151-152. Sheldrick, G. M. (2008). Acta Cryst. A64, 112-122. 


\title{
supporting information
}

Acta Cryst. (2011). E67, o2239 [doi:10.1107/S1600536811028868]

\section{Bis[N-cyclohexyl-1-(2-\{1-[(cyclohexylamino)carbonyl]cyclohexyl\}-3,5- dioxo-1,2-oxazolidin-4-yl)cyclopentanecarboxamide] monohydrate}

\author{
Enayatollah Sheikhhosseini, Fahime Vafadarnejad and Azizollah Habibi
}

\section{S1. Comment}

Isoxazoles play an important role in heterocyclic chemistry and have served as versatile building blocks in organic synthesis. They have long been targeted in synthetic investigations for their known biological activities and pharmacological properties such as hypoglycemic, analgesic, anti-inflammatory and anti-bacterial activities (Conti et al., 1998; Mishra et al., 1998; Ko et al., 1998; Kang et al., 2000). The title compound was formed by reaction of cyclohexyl isocyanide and alkylidene Meldrum's acid in the presence of cyclohexyl ketoxime in dichloromethane as solvent. Fig. 1 shows the crystal structure of the title compound. The cyclohexyl and cyclopentyl rings have the normal shape (chair conformation for cyclohexyl and twisted envelope conformation for cyclopentyl ring), bond lengths and angles. Their planes align almost perpendicular in respect to the isoxazole core. The crystal structure is stabilized by $\mathrm{N}-\mathrm{H} \cdots \mathrm{O}$ and $\mathrm{O}$ $-\mathrm{H} \cdots \mathrm{O}$ hydrogen bonds (Table 1, Fig. 2). Both molecules in the asymmetric unit interact by N3B-H3BA $\cdots \mathrm{O} 3 \mathrm{~A}$ hydrogen bonding with $\mathrm{D} \cdots \mathrm{A}$ distance of 2.9238 (17) $\AA$. There is also a N2B-H2BA $\cdots \mathrm{O} 5 \mathrm{~B}$ intermolecular hydrogen bonding with $\mathrm{D} \cdots \mathrm{A}$ distance of 2.9330 (18) $\AA$. The water molecule connects the molecules by N3A-H3AA $\cdots \mathrm{O} 1 \mathrm{~W}, \mathrm{O} 1 \mathrm{~W}$ $-\mathrm{H} 1 \mathrm{WA} \cdots \mathrm{O} 4 \mathrm{~B}(-x+1,-y+2,-z+1)$ and $\mathrm{O} 1 \mathrm{~W}-\mathrm{H} 1 \mathrm{WB} \cdots \mathrm{O} 4 \mathrm{~A}(x+1, y, z)$ hydrogen bonds with $\mathrm{D} \cdots \mathrm{A}$ distances of 2.8662 (19), 2.7433 (18) and 2.7569 (18) Å respectively.

\section{S2. Experimental}

The title compound was prepared by reaction of cyclohexyl isocyanide ( $4 \mathrm{mmol}, 0.439 \mathrm{~g})$ and alkylidene Meldrum's acid $(2 \mathrm{mmol}, 0.421 \mathrm{~g})$ in the presence of cyclohexyl ketoxime $(2 \mathrm{mmol}, 0.226 \mathrm{~g})$ in dichloromethane at room temperature within four hours. After evaporating the solvent, a white powder was separated from the reaction mixture.Crystallization and single-crystal preparation was done from an ethanol solution. Colorless crystals were obtained after two weeks at room temperature.

\section{S3. Refinement}

The hydrogen atoms of the water molecule and $\mathrm{NH}$ groups were found in a difference Fourier synthesis. The hydrogen atoms of the water molecule were refined in an isotropic approximation. The other hydrogen atoms were refined using a riding model with the $U_{\text {iso }}(\mathrm{H})$ parameters equal to $1.2 U_{\text {eq }}(\mathrm{Ci}, \mathrm{Nj})$ and $\mathrm{C}-\mathrm{H}=0.99$ and $1.00 \AA$, and $\mathrm{N}-\mathrm{H}=0.88 \AA$. In molecule $\mathrm{B}$, atom $\mathrm{C} 19$ is disordered over two positions with occupancies $0.75 / 0.25$. 


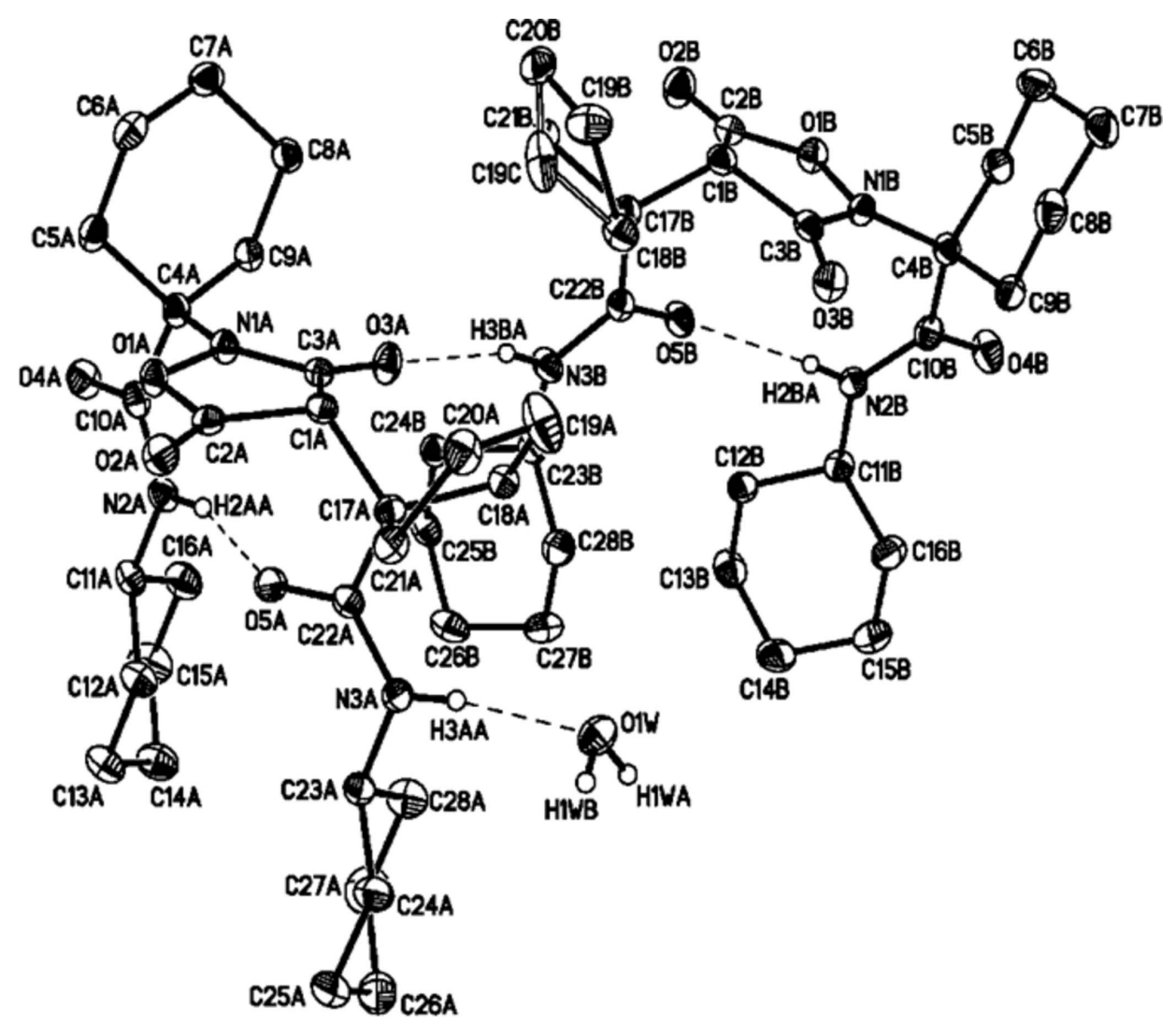

\section{Figure 1}

Molecular structure of the title compound with thermal elliposids drawn at 50\% probability level. Hydrogen bonds are shown as dashed lines. Hydrogen atoms not involved inhydrogen bonds have been omitted for clarity. 


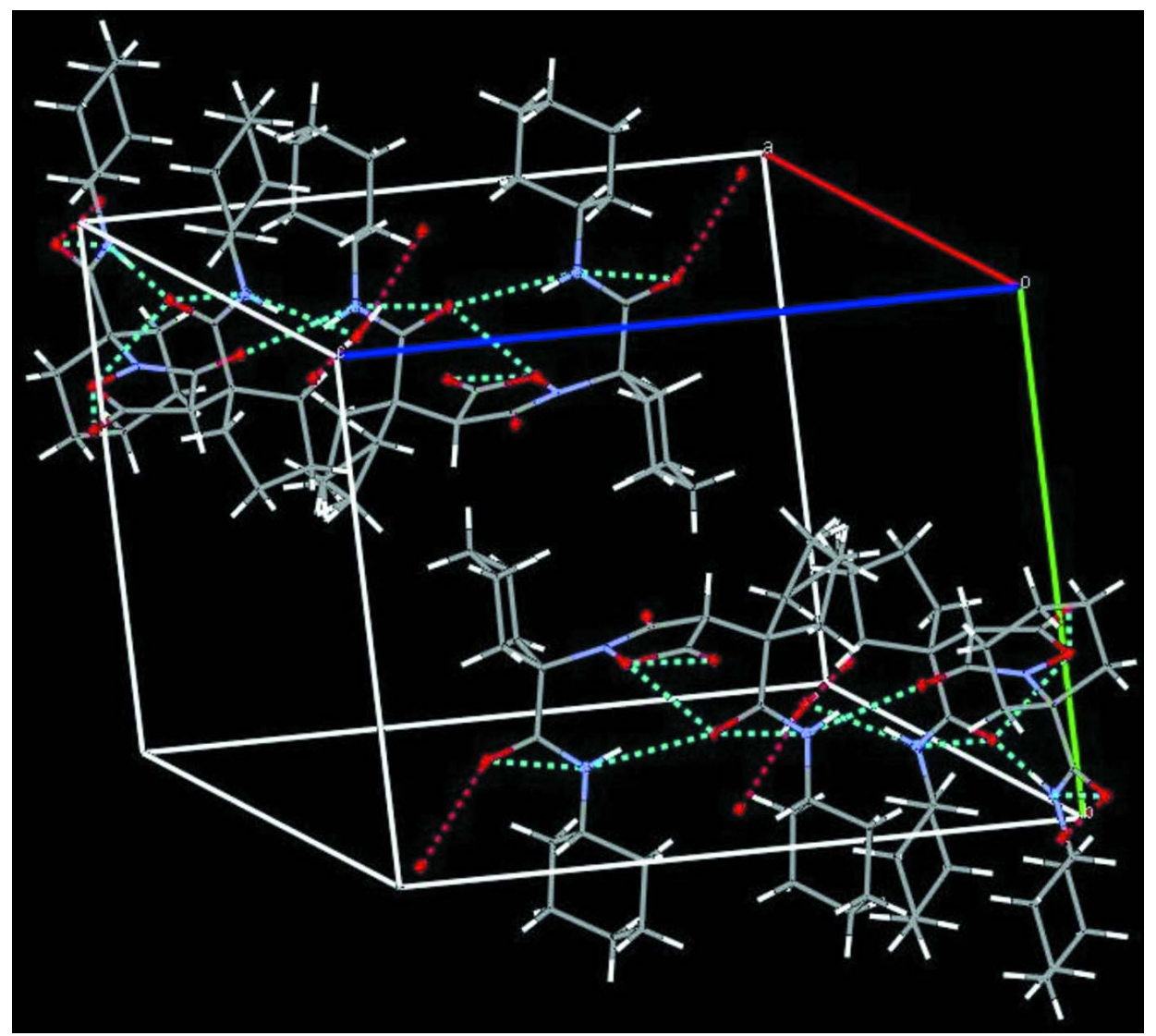

Figure 2

Unit cell packing diagram of the title compound. Hydrogen bonds are shown as dashed lines.

Bis[N-Cyclohexyl-1-(2-\{1-[(cyclohexylamino)carbonyl]cyclohexyl\}-3,5- dioxo-1,2-oxazolidin-4yl)cyclopentanecarboxamide] monohydrate

\section{Crystal data}

$2 \mathrm{C}_{28} \mathrm{H}_{43} \mathrm{~N}_{3} \mathrm{O}_{5} \cdot \mathrm{H}_{2} \mathrm{O}$

$M_{r}=1021.32$

Triclinic, $P \overline{1}$

Hall symbol: -P 1

$a=11.3084(6) \AA$

$b=12.3119(7) \AA$

$c=20.7904(11) \AA$

$\alpha=102.686(1)^{\circ}$

$\beta=103.104(1)^{\circ}$

$\gamma=93.100(1)^{\circ}$

$V=2733.9(3) \AA^{3}$

Data collection

\section{Bruker APEXII CCD}

diffractometer

Radiation source: fine-focus sealed tube

Graphite monochromator

$\varphi$ and $\omega$ scans
$Z=2$

$F(000)=1108$

$D_{\mathrm{x}}=1.241 \mathrm{Mg} \mathrm{m}^{-3}$

Mo $K \alpha$ radiation, $\lambda=0.71073 \AA$

Cell parameters from 7443 reflections

$\theta=2.3-30.5^{\circ}$

$\mu=0.09 \mathrm{~mm}^{-1}$

$T=100 \mathrm{~K}$

Plate, colourless

$0.60 \times 0.50 \times 0.10 \mathrm{~mm}$

28848 measured reflections

11931 independent reflections

9099 reflections with $I>2 \sigma(I)$

$R_{\text {int }}=0.028$

$\theta_{\max }=27.0^{\circ}, \theta_{\min }=1.8^{\circ}$ 
$h=-14 \rightarrow 14$

$k=-15 \rightarrow 15$

\section{Refinement}

Refinement on $F^{2}$

Least-squares matrix: full

$R\left[F^{2}>2 \sigma\left(F^{2}\right)\right]=0.047$

$w R\left(F^{2}\right)=0.120$

$S=1.03$

11931 reflections

675 parameters

0 restraints

Primary atom site location: structure-invariant direct methods $l=-26 \rightarrow 26$

Secondary atom site location: difference Fourier map

Hydrogen site location: mixed

$\mathrm{H}$ atoms treated by a mixture of independent and constrained refinement

$w=1 /\left[\sigma^{2}\left(F_{\mathrm{o}}^{2}\right)+(0.0534 P)^{2}+1.1858 P\right]$

where $P=\left(F_{\mathrm{o}}^{2}+2 F_{\mathrm{c}}{ }^{2}\right) / 3$

$(\Delta / \sigma)_{\max }=0.001$

$\Delta \rho_{\max }=0.38 \mathrm{e} \AA^{-3}$

$\Delta \rho_{\min }=-0.25$ e $\AA^{-3}$

\section{Special details}

Geometry. All e.s.d.'s (except the e.s.d. in the dihedral angle between two 1.s. planes) are estimated using the full covariance matrix. The cell e.s.d.'s are taken into account individually in the estimation of e.s.d.'s in distances, angles and torsion angles; correlations between e.s.d.'s in cell parameters are only used when they are defined by crystal symmetry. An approximate (isotropic) treatment of cell e.s.d.'s is used for estimating e.s.d.'s involving 1.s. planes.

Refinement. Refinement of $F^{2}$ against ALL reflections. The weighted $R$-factor $w R$ and goodness of fit $S$ are based on $F^{2}$, conventional $R$-factors $R$ are based on $F$, with $F$ set to zero for negative $F^{2}$. The threshold expression of $F^{2}>\sigma\left(F^{2}\right)$ is used only for calculating $R$-factors(gt) etc. and is not relevant to the choice of reflections for refinement. $R$-factors based on $F^{2}$ are statistically about twice as large as those based on $F$, and $R$ - factors based on ALL data will be even larger.

Fractional atomic coordinates and isotropic or equivalent isotropic displacement parameters $\left(\hat{A}^{2}\right)$

\begin{tabular}{llllll}
\hline & $x$ & $y$ & $z$ & $U_{\text {iso }} / U_{\text {eq }}$ & Occ. $(<1)$ \\
\hline O1A & $-0.13320(10)$ & $0.65250(9)$ & $0.03789(5)$ & $0.0176(2)$ & \\
O2A & $0.01027(10)$ & $0.61354(9)$ & $-0.01804(5)$ & $0.0219(2)$ & \\
O3A & $-0.00419(10)$ & $0.72134(10)$ & $0.21203(5)$ & $0.0223(3)$ & \\
O4A & $-0.39218(10)$ & $0.84717(10)$ & $0.09932(6)$ & $0.0248(3)$ & \\
O5A & $0.05691(10)$ & $0.85482(9)$ & $0.09712(6)$ & $0.0195(2)$ & \\
N1A & $-0.13848(12)$ & $0.67986(11)$ & $0.10777(6)$ & $0.0161(3)$ & \\
N2A & $-0.19391(12)$ & $0.89376(11)$ & $0.10505(7)$ & $0.0193(3)$ & \\
H2AA & -0.1201 & 0.8723 & 0.1095 & $0.023^{*}$ & \\
N3A & $0.25882(12)$ & $0.91251(11)$ & $0.13761(7)$ & $0.0190(3)$ & \\
H3AA & 0.3303 & 0.8948 & 0.1572 & $0.023^{*}$ & \\
C1A & $0.06124(14)$ & $0.64695(12)$ & $0.10753(8)$ & $0.0154(3)$ & \\
H1AA & 0.0758 & 0.5696 & 0.1127 & $0.018^{*}$ & \\
C2A & $-0.01422(14)$ & $0.63683(12)$ & $0.03600(8)$ & $0.0165(3)$ & \\
C3A & $-0.02645(14)$ & $0.68957(12)$ & $0.15035(8)$ & $0.0159(3)$ & \\
C4A & $-0.26030(14)$ & $0.70536(13)$ & $0.11588(8)$ & $0.0172(3)$ & \\
C5A & $-0.35683(14)$ & $0.61647(14)$ & $0.06433(8)$ & $0.0200(3)$ & \\
H5AA & -0.4393 & 0.6368 & 0.0672 & $0.024^{*}$ & \\
H5AB & -0.3486 & 0.6155 & 0.0178 & $0.024^{*}$ & \\
C6A & $-0.34373(15)$ & $0.49955(14)$ & $0.07719(9)$ & $0.0234(4)$ & \\
H6AA & -0.4092 & 0.4455 & 0.0440 & $0.028^{*}$ & \\
H6AB & -0.2643 & 0.4763 & 0.0702 & $0.028^{*}$ \\
C7A & $-0.35121(15)$ & $0.49669(14)$ & $0.14915(9)$ & $0.0236(4)$ &
\end{tabular}




\begin{tabular}{|c|c|c|c|c|}
\hline H7AA & -0.4344 & 0.5102 & 0.1544 & $0.028 *$ \\
\hline $\mathrm{H} 7 \mathrm{AB}$ & -0.3357 & 0.4216 & 0.1569 & $0.028 *$ \\
\hline $\mathrm{C} 8 \mathrm{~A}$ & $-0.25824(14)$ & 0.58504 (14) & $0.20171(8)$ & $0.0203(3)$ \\
\hline H8AA & -0.1749 & 0.5650 & 0.2009 & $0.024 *$ \\
\hline H8AB & -0.2701 & 0.5860 & 0.2476 & $0.024 *$ \\
\hline C9A & $-0.26971(14)$ & $0.70175(13)$ & $0.18846(8)$ & 0.0179 \\
\hline H9AA & -0.2044 & 0.7554 & 0.2220 & $0.021^{*}$ \\
\hline H9AB & -0.3493 & 0.7256 & 0.1950 & $0.021^{*}$ \\
\hline C10A & $-0.28653(14)$ & $0.82245(13)$ & $0.10540(8)$ & $0.0187(3)$ \\
\hline C11A & $-0.21330(15)$ & $1.00748(13)$ & $0.09738(8)$ & $0.0201(3)$ \\
\hline H11A & -0.2916 & 1.0034 & 0.0625 & $0.024^{*}$ \\
\hline C12A & $-0.11029(16)$ & $1.05422(14)$ & $0.07207(9)$ & $0.0230(3)$ \\
\hline H12A & -0.1073 & 1.0048 & 0.0281 & $0.028^{*}$ \\
\hline H12B & -0.0315 & 1.0555 & 0.1050 & $0.028^{*}$ \\
\hline $\mathrm{C} 13 \mathrm{~A}$ & $-0.12769(18)$ & $1.17244(14)$ & $0.06302(9)$ & $0.0278(4)$ \\
\hline H13A & -0.0572 & 1.2021 & 0.0488 & $0.033^{*}$ \\
\hline H13B & -0.2022 & 1.1701 & 0.0267 & $0.033^{*}$ \\
\hline C14A & $-0.13910(18)$ & $1.24998(15)$ & $0.12894(10)$ & 0.0307 (4) \\
\hline H14A & -0.1558 & 1.3247 & 0.1210 & $0.037^{*}$ \\
\hline H14B & -0.0611 & 1.2591 & 0.1638 & $0.037^{*}$ \\
\hline $\mathrm{C} 15 \mathrm{~A}$ & -0.24185 (19) & $1.20278(15)$ & $0.15483(10)$ & $0.0335(4)$ \\
\hline H15A & -0.3210 & 1.2012 & 0.1221 & $0.040^{*}$ \\
\hline H15B & -0.2445 & 1.2522 & 0.1989 & $0.040^{*}$ \\
\hline $\mathrm{C} 16 \mathrm{~A}$ & $-0.22361(17)$ & $1.08438(15)$ & $0.16395(9)$ & $0.0269(4)$ \\
\hline H16A & -0.1484 & 1.0868 & 0.1998 & $0.032 *$ \\
\hline H16B & -0.2935 & 1.0544 & 0.1786 & $0.032 *$ \\
\hline C17A & $0.18704(13)$ & $0.71710(12)$ & $0.12690(8)$ & $0.0156(3)$ \\
\hline C18A & $0.26142(14)$ & $0.70717(13)$ & $0.19718(8)$ & 0.0183 \\
\hline H18A & 0.3270 & 0.7702 & 0.2171 & $0.022 *$ \\
\hline H18B & 0.2079 & 0.7072 & 0.2288 & $0.022 *$ \\
\hline C19A & $0.3158(2)$ & $0.59538(17)$ & $0.18350(10)$ & $0.0363(5)$ \\
\hline H19A & 0.2692 & 0.5375 & 0.1970 & $0.044 *$ \\
\hline H19B & 0.4020 & 0.6038 & 0.2096 & $0.044 *$ \\
\hline $\mathrm{C} 20 \mathrm{~A}$ & $0.30729(15)$ & $0.56189(14)$ & $0.10647(9)$ & $0.0236(4)$ \\
\hline $\mathrm{H} 20 \mathrm{~A}$ & 0.3873 & 0.5435 & 0.0978 & $0.028 *$ \\
\hline H20B & 0.2460 & 0.4962 & 0.0839 & $0.028^{*}$ \\
\hline $\mathrm{C} 21 \mathrm{~A}$ & $0.26873(14)$ & $0.66455(13)$ & $0.08054(8)$ & $0.0187(3)$ \\
\hline $\mathrm{H} 21 \mathrm{~A}$ & 0.2224 & 0.6426 & 0.0323 & $0.022 *$ \\
\hline $\mathrm{H} 21 \mathrm{~B}$ & 0.3408 & 0.7175 & 0.0849 & $0.022^{*}$ \\
\hline $\mathrm{C} 22 \mathrm{~A}$ & $0.16267(14)$ & $0.83580(13)$ & $0.12053(8)$ & $0.0165(3)$ \\
\hline $\mathrm{C} 23 \mathrm{~A}$ & $0.25175(15)$ & $1.02484(13)$ & $0.12546(8)$ & $0.0189(3)$ \\
\hline $\mathrm{H} 23 \mathrm{~A}$ & 0.1742 & 1.0236 & 0.0906 & $0.023^{*}$ \\
\hline $\mathrm{C} 24 \mathrm{~A}$ & $0.35833(16)$ & 1.05665 (14) & $0.09700(9)$ & $0.0242(4)$ \\
\hline $\mathrm{H} 24 \mathrm{~B}$ & 0.4363 & 1.0576 & 0.1305 & $0.029^{*}$ \\
\hline $\mathrm{H} 24 \mathrm{C}$ & 0.3578 & 1.0000 & 0.0549 & $0.029 *$ \\
\hline $\mathrm{C} 25 \mathrm{~A}$ & $0.34905(18)$ & $1.17178(15)$ & $0.08134(9)$ & $0.0314(4)$ \\
\hline $\mathrm{H} 25 \mathrm{~A}$ & 0.4204 & 1.1923 & 0.0646 & $0.038^{*}$ \\
\hline H25B & 0.2744 & 1.1691 & 0.0451 & $0.038^{*}$ \\
\hline
\end{tabular}




\begin{tabular}{|c|c|c|c|c|}
\hline $\mathrm{C} 26 \mathrm{~A}$ & $0.34470(18)$ & $1.26019(15)$ & $0.14446(10)$ & $0.0338(4)$ \\
\hline $\mathrm{H} 26 \mathrm{~A}$ & 0.4235 & 1.2692 & 0.1787 & $0.041^{*}$ \\
\hline $\mathrm{H} 26 \mathrm{~B}$ & 0.3328 & 1.3329 & 0.1323 & $0.041^{*}$ \\
\hline $\mathrm{C} 27 \mathrm{~A}$ & $0.24173(19)$ & $1.22803(15)$ & $0.17496(11)$ & $0.0361(5)$ \\
\hline $\mathrm{H} 27 \mathrm{~A}$ & 0.1624 & 1.2289 & 0.1430 & $0.043^{*}$ \\
\hline H27B & 0.2455 & 1.2841 & 0.2178 & $0.043^{*}$ \\
\hline $\mathrm{C} 28 \mathrm{~A}$ & $0.24952(17)$ & $1.11198(14)$ & $0.18959(9)$ & $0.0269(4)$ \\
\hline $\mathrm{H} 28 \mathrm{~A}$ & 0.3245 & 1.1131 & 0.2254 & $0.032 *$ \\
\hline $\mathrm{H} 28 \mathrm{~B}$ & 0.1783 & 1.0918 & 0.2065 & $0.032 *$ \\
\hline O1B & $0.16762(10)$ & $0.67637(9)$ & $0.57514(6)$ & $0.0204(2)$ \\
\hline $\mathrm{O} 2 \mathrm{~B}$ & $-0.02284(11)$ & $0.63046(11)$ & $0.51393(6)$ & $0.0282(3)$ \\
\hline $\mathrm{O} 3 \mathrm{~B}$ & $0.36665(10)$ & $0.65175(10)$ & $0.46859(6)$ & $0.0238(3)$ \\
\hline O4B & $0.43163(12)$ & $0.91008(10)$ & $0.70259(6)$ & $0.0305(3)$ \\
\hline $\mathrm{O} 5 \mathrm{~B}$ & $0.14185(11)$ & $0.81491(9)$ & $0.47007(5)$ & $0.0231(3)$ \\
\hline N1B & $0.28750(11)$ & $0.67806(11)$ & $0.56210(6)$ & $0.0171(3)$ \\
\hline $\mathrm{N} 2 \mathrm{~B}$ & $0.32941(12)$ & $0.91170(11)$ & $0.59588(7)$ & $0.0189(3)$ \\
\hline $\mathrm{H} 2 \mathrm{BA}$ & 0.2902 & 0.8715 & 0.5554 & $0.023 *$ \\
\hline N3B & $0.07309(13)$ & $0.81334(11)$ & $0.35903(7)$ & $0.0213(3)$ \\
\hline H3BA & 0.0514 & 0.7721 & 0.3171 & $0.026^{*}$ \\
\hline $\mathrm{C} 1 \mathrm{~B}$ & $0.14912(15)$ & $0.60000(13)$ & $0.45886(8)$ & $0.0187(3)$ \\
\hline H1BA & 0.1448 & 0.5164 & 0.4483 & $0.022 *$ \\
\hline $\mathrm{C} 2 \mathrm{~B}$ & $0.08379(15)$ & $0.63657(13)$ & $0.51466(8)$ & $0.0196(3)$ \\
\hline $\mathrm{C} 3 \mathrm{~B}$ & $0.28102(14)$ & $0.64675(13)$ & $0.49443(8)$ & $0.0177(3)$ \\
\hline C4B & $0.38224(14)$ & $0.73222(13)$ & $0.62359(8)$ & $0.0176(3)$ \\
\hline $\mathrm{C} 5 \mathrm{~B}$ & $0.36582(16)$ & $0.68078(14)$ & $0.68243(8)$ & $0.0236(4)$ \\
\hline H5BA & 0.4218 & 0.7245 & 0.7253 & $0.028^{*}$ \\
\hline H5BB & 0.2811 & 0.6859 & 0.6874 & $0.028^{*}$ \\
\hline C6B & $0.39141(18)$ & $0.55844(15)$ & $0.67121(9)$ & 0.0305 (4) \\
\hline H6BA & 0.3836 & 0.5303 & 0.7113 & $0.037^{*}$ \\
\hline H6BB & 0.3300 & 0.5131 & 0.6311 & $0.037^{*}$ \\
\hline $\mathrm{C} 7 \mathrm{~B}$ & $0.51869(17)$ & $0.54440(15)$ & $0.66002(9)$ & 0.0309 (4) \\
\hline H7BA & 0.5806 & 0.5837 & 0.7017 & $0.037 *$ \\
\hline H7BB & 0.5307 & 0.4639 & 0.6508 & $0.037 *$ \\
\hline $\mathrm{C} 8 \mathrm{~B}$ & $0.53603(16)$ & $0.59126(15)$ & $0.60090(9)$ & $0.0268(4)$ \\
\hline H8BA & 0.4804 & 0.5463 & 0.5583 & $0.032 *$ \\
\hline H8BB & 0.6210 & 0.5857 & 0.5965 & $0.032 *$ \\
\hline C9B & $0.51007(15)$ & $0.71315(14)$ & $0.61091(8)$ & $0.0215(3)$ \\
\hline H9BA & 0.5170 & 0.7393 & 0.5700 & $0.026^{*}$ \\
\hline H9BB & 0.5729 & 0.7591 & 0.6501 & $0.026^{*}$ \\
\hline $\mathrm{C} 10 \mathrm{~B}$ & $0.37977(15)$ & $0.86003(14)$ & $0.64358(8)$ & $0.0204(3)$ \\
\hline C11B & $0.33796(15)$ & $1.03416(13)$ & $0.60937(8)$ & $0.0196(3)$ \\
\hline H11B & 0.3238 & 1.0636 & 0.6555 & $0.024 *$ \\
\hline $\mathrm{C} 12 \mathrm{~B}$ & $0.23958(15)$ & $1.06971(13)$ & $0.55735(8)$ & 0.0209 (3) \\
\hline $\mathrm{H} 12 \mathrm{C}$ & 0.1581 & 1.0390 & 0.5594 & $0.025^{*}$ \\
\hline H12D & 0.2503 & 1.0391 & 0.5111 & $0.025^{*}$ \\
\hline C13B & $0.24687(17)$ & $1.19743(14)$ & $0.57150(9)$ & $0.0284(4)$ \\
\hline $\mathrm{H} 13 \mathrm{C}$ & 0.1835 & 1.2195 & 0.5368 & $0.034^{*}$ \\
\hline H13D & 0.2312 & 1.2278 & 0.6166 & $0.034 *$ \\
\hline
\end{tabular}




\begin{tabular}{|c|c|c|c|c|c|}
\hline C14B & $0.37301(19)$ & $1.24610(15)$ & $0.56991(10)$ & $0.0351(4)$ & \\
\hline $\mathrm{H} 14 \mathrm{C}$ & 0.3860 & 1.2198 & 0.5238 & $0.042 *$ & \\
\hline H14D & 0.3774 & 1.3288 & 0.5803 & $0.042 *$ & \\
\hline C15B & $0.47295(18)$ & $1.21077(15)$ & $0.62142(11)$ & $0.0349(4)$ & \\
\hline $\mathrm{H} 15 \mathrm{C}$ & 0.4647 & 1.2432 & 0.6680 & $0.042 *$ & \\
\hline H15D & 0.5539 & 1.2400 & 0.6179 & $0.042 *$ & \\
\hline C16B & $0.46468(16)$ & $1.08332(14)$ & $0.60890(10)$ & $0.0271(4)$ & \\
\hline $\mathrm{H} 16 \mathrm{C}$ & 0.5269 & 1.0624 & 0.6446 & $0.032 *$ & \\
\hline H16D & 0.4822 & 1.0515 & 0.5644 & $0.032 *$ & \\
\hline C17B & $0.09761(14)$ & $0.63456(13)$ & $0.39177(8)$ & 0.0178 & \\
\hline C18B & $0.16391(16)$ & $0.58323(13)$ & $0.33668(8)$ & $0.0222(3)$ & \\
\hline $\mathrm{H} 18 \mathrm{C}$ & 0.2496 & 0.5840 & 0.3561 & $0.027 *$ & \\
\hline H18D & 0.1538 & 0.6238 & 0.3016 & $0.027^{*}$ & \\
\hline C19B & 0.1021 & $0.4634(2)$ & $0.30824(15)$ & $0.0298(6)$ & 0.75 \\
\hline $\mathrm{H} 19 \mathrm{C}$ & 0.0970 & 0.4395 & 0.2589 & $0.036^{*}$ & 0.75 \\
\hline H19D & 0.1493 & 0.4115 & 0.3314 & $0.036^{*}$ & 0.75 \\
\hline C19C & $0.0770(8)$ & $0.4970(7)$ & $0.2808(4)$ & $0.034(2)$ & 0.25 \\
\hline H19E & 0.1184 & 0.4318 & 0.2637 & $0.040 *$ & 0.25 \\
\hline $\mathrm{H} 19 \mathrm{~F}$ & 0.0392 & 0.5293 & 0.2425 & $0.040 *$ & 0.25 \\
\hline $\mathrm{C} 20 \mathrm{~B}$ & $-0.02488(16)$ & $0.46242(14)$ & $0.32074(10)$ & $0.0288(4)$ & \\
\hline $\mathrm{H} 20 \mathrm{C}$ & -0.0362 & 0.4100 & 0.3474 & $0.035^{*}$ & \\
\hline $\mathrm{H} 20 \mathrm{D}$ & -0.0856 & 0.4416 & 0.2783 & $0.035 *$ & \\
\hline $\mathrm{C} 21 \mathrm{~B}$ & $-0.03579(15)$ & $0.58055(14)$ & $0.35886(8)$ & $0.0223(3)$ & \\
\hline $\mathrm{H} 21 \mathrm{C}$ & -0.0812 & 0.5786 & 0.3942 & $0.027^{*}$ & \\
\hline $\mathrm{H} 21 \mathrm{D}$ & -0.0788 & 0.6232 & 0.3272 & $0.027 *$ & \\
\hline $\mathrm{C} 22 \mathrm{~B}$ & $0.10709(14)$ & $0.76261(13)$ & $0.40977(8)$ & $0.0182(3)$ & \\
\hline $\mathrm{C} 23 \mathrm{~B}$ & $0.07040(14)$ & $0.93452(13)$ & $0.37026(8)$ & $0.0182(3)$ & \\
\hline $\mathrm{H} 23 \mathrm{~B}$ & 0.0716 & 0.9650 & 0.4192 & $0.022 *$ & \\
\hline $\mathrm{C} 24 \mathrm{~B}$ & $-0.04667(15)$ & $0.96237(13)$ & $0.32703(8)$ & $0.0197(3)$ & \\
\hline $\mathrm{H} 24 \mathrm{~A}$ & -0.1184 & 0.9236 & 0.3357 & $0.024^{*}$ & \\
\hline H24D & -0.0485 & 0.9362 & 0.2782 & $0.024 *$ & \\
\hline $\mathrm{C} 25 \mathrm{~B}$ & $-0.05234(16)$ & $1.08885(14)$ & $0.34468(9)$ & $0.0233(4)$ & \\
\hline $\mathrm{H} 25 \mathrm{C}$ & -0.1285 & 1.1070 & 0.3167 & $0.028 *$ & \\
\hline $\mathrm{H} 25 \mathrm{D}$ & -0.0534 & 1.1143 & 0.3931 & $0.028^{*}$ & \\
\hline $\mathrm{C} 26 \mathrm{~B}$ & $0.05742(17)$ & $1.14980(14)$ & $0.33163(9)$ & $0.0282(4)$ & \\
\hline $\mathrm{H} 26 \mathrm{C}$ & 0.0551 & 1.2316 & 0.3462 & $0.034 *$ & \\
\hline $\mathrm{H} 26 \mathrm{D}$ & 0.0530 & 1.1308 & 0.2823 & $0.034 *$ & \\
\hline $\mathrm{C} 27 \mathrm{~B}$ & $0.17706(17)$ & $1.11818(15)$ & $0.36985(10)$ & $0.0300(4)$ & \\
\hline $\mathrm{H} 27 \mathrm{C}$ & 0.1877 & 1.1485 & 0.4193 & $0.036^{*}$ & \\
\hline $\mathrm{H} 27 \mathrm{D}$ & 0.2457 & 1.1529 & 0.3564 & $0.036^{*}$ & \\
\hline C28B & $0.18147(15)$ & $0.99154(14)$ & $0.35580(9)$ & $0.0251(4)$ & \\
\hline $\mathrm{H} 28 \mathrm{C}$ & 0.1829 & 0.9621 & 0.3077 & $0.030 *$ & \\
\hline $\mathrm{H} 28 \mathrm{D}$ & 0.2571 & 0.9746 & 0.3849 & $0.030 *$ & \\
\hline $\mathrm{O} 1 \mathrm{~W}$ & $0.50041(11)$ & $0.88475(10)$ & $0.20760(7)$ & $0.0232(3)$ & \\
\hline H1WA & $0.531(2)$ & $0.946(2)$ & $0.2367(13)$ & $0.053(7)^{*}$ & \\
\hline H1WB & $0.540(2)$ & $0.881(2)$ & 0.1749 (13) & $0.053(7)^{*}$ & \\
\hline
\end{tabular}


Atomic displacement parameters $\left(\AA^{2}\right)$

\begin{tabular}{|c|c|c|c|c|c|c|}
\hline & $U^{11}$ & $U^{22}$ & $U^{33}$ & $U^{12}$ & $U^{13}$ & $U^{23}$ \\
\hline O1A & $0.0175(5)$ & $0.0229(6)$ & $0.0116(5)$ & $0.0020(4)$ & $0.0033(4)$ & $0.0031(4)$ \\
\hline $\mathrm{O} 2 \mathrm{~A}$ & $0.0243(6)$ & $0.0244(6)$ & $0.0163(6)$ & $0.0006(5)$ & $0.0067(5)$ & $0.0020(5)$ \\
\hline $\mathrm{O} 3 \mathrm{~A}$ & $0.0181(6)$ & $0.0337(7)$ & $0.0131(5)$ & $0.0012(5)$ & $0.0030(4)$ & $0.0029(5)$ \\
\hline O4A & $0.0194(6)$ & $0.0300(7)$ & $0.0287(6)$ & $0.0085(5)$ & $0.0076(5)$ & $0.0114(5)$ \\
\hline O5A & $0.0164(5)$ & $0.0186(6)$ & $0.0246(6)$ & 0.0033 & $0.0043(5)$ & $0.0075(5)$ \\
\hline N1A & $0.0168(6)$ & $0.0200(7)$ & $0.0119(6)$ & $0.0024(5)$ & $0.0047(5)$ & $0.0032(5)$ \\
\hline $\mathrm{N} 2 \mathrm{~A}$ & $0.0185(7)$ & $0.0204(7)$ & $0.0212(7)$ & $0.0060(5)$ & $0.0052(5)$ & $0.0081(6)$ \\
\hline N3A & $0.0164(6)$ & $0.0171(7)$ & $0.0233(7)$ & $0.0016(5)$ & $0.0020(5)$ & $0.0075(5)$ \\
\hline C1A & $0.0172(7)$ & $0.0126(7)$ & $0.0169(7)$ & $0.0022(6)$ & 0.0048 (6) & $0.0039(6)$ \\
\hline $\mathrm{C} 2 \mathrm{~A}$ & $0.0175(7)$ & $0.0124(7)$ & $0.0191(8)$ & $0.0000(6)$ & $0.0036(6)$ & $0.0039(6)$ \\
\hline C3A & $0.0156(7)$ & $0.0150(7)$ & $0.0176(8)$ & $0.0008(6)$ & $0.0036(6)$ & $0.0058(6)$ \\
\hline $\mathrm{C} 4 \mathrm{~A}$ & $0.0132(7)$ & $0.0219(8)$ & $0.0169(8)$ & $0.0036(6)$ & $0.0036(6)$ & $0.0054(6)$ \\
\hline C5A & $0.0149(7)$ & $0.0258(8)$ & $0.0178(8)$ & $0.0006(6)$ & $0.0023(6)$ & $0.0042(6)$ \\
\hline C6A & $0.0175(8)$ & $0.0231(8)$ & 0.0257 (9) & $-0.0007(6)$ & $0.0020(7)$ & $0.0017(7)$ \\
\hline C7A & $0.0172(8)$ & $0.0223(8)$ & $0.0316(9)$ & $0.0007(6)$ & $0.0041(7)$ & $0.0093(7)$ \\
\hline C8A & $0.0170(8)$ & $0.0256(8)$ & $0.0204(8)$ & $0.0021(6)$ & $0.0046(6)$ & $0.0095(7)$ \\
\hline C9A & $0.0157(7)$ & $0.0222(8)$ & $0.0169(8)$ & $0.0032(6)$ & $0.0052(6)$ & $0.0058(6)$ \\
\hline C10A & $0.0193(8)$ & $0.0238(8)$ & $0.0142(7)$ & $0.0053(6)$ & 0.0047 (6) & $0.0055(6)$ \\
\hline C11A & $0.0234(8)$ & $0.0202(8)$ & $0.0185(8)$ & $0.0066(6)$ & $0.0051(7)$ & $0.0074(6)$ \\
\hline C12A & $0.0292(9)$ & $0.0212(8)$ & $0.0223(8)$ & $0.0060(7)$ & $0.0108(7)$ & $0.0075(7)$ \\
\hline C13A & $0.0364(10)$ & $0.0241(9)$ & $0.0283(9)$ & $0.0079(8)$ & $0.0118(8)$ & $0.0125(7)$ \\
\hline C14A & $0.0404(11)$ & $0.0197(9)$ & $0.0355(10)$ & $0.0061(8)$ & $0.0138(9)$ & $0.0084(8)$ \\
\hline $\mathrm{C} 15 \mathrm{~A}$ & $0.0458(12)$ & $0.0236(9)$ & $0.0357(10)$ & $0.0109(8)$ & $0.0204(9)$ & $0.0043(8)$ \\
\hline C16A & $0.0354(10)$ & $0.0263(9)$ & $0.0220(9)$ & $0.0055(8)$ & $0.0122(8)$ & $0.0059(7)$ \\
\hline C17A & $0.0145(7)$ & $0.0161(7)$ & $0.0167(7)$ & $0.0013(6)$ & $0.0049(6)$ & $0.0039(6)$ \\
\hline C18A & $0.0169(7)$ & $0.0200(8)$ & $0.0179(8)$ & $0.0005(6)$ & $0.0031(6)$ & $0.0059(6)$ \\
\hline C19A & $0.0468(12)$ & $0.0377(11)$ & $0.0269(10)$ & $0.0226(9)$ & $0.0064(9)$ & $0.0111(8)$ \\
\hline $\mathrm{C} 20 \mathrm{~A}$ & $0.0202(8)$ & $0.0234(9)$ & $0.0274(9)$ & $0.0077(7)$ & 0.0058 & $0.0055(7)$ \\
\hline $\mathrm{C} 21 \mathrm{~A}$ & $0.0161(7)$ & $0.0206(8)$ & $0.0205(8)$ & $0.0037(6)$ & $0.0069(6)$ & $0.0042(6)$ \\
\hline $\mathrm{C} 22 \mathrm{~A}$ & $0.0190(8)$ & $0.0178(8)$ & $0.0141(7)$ & $0.0031(6)$ & $0.0066(6)$ & $0.0040(6)$ \\
\hline $\mathrm{C} 23 \mathrm{~A}$ & $0.0203(8)$ & $0.0158(7)$ & $0.0201(8)$ & $0.0012(6)$ & $0.0026(6)$ & $0.0056(6)$ \\
\hline $\mathrm{C} 24 \mathrm{~A}$ & $0.0281(9)$ & $0.0227(8)$ & $0.0236(9)$ & $-0.0007(7)$ & $0.0091(7)$ & $0.0071(7)$ \\
\hline $\mathrm{C} 25 \mathrm{~A}$ & $0.0360(10)$ & $0.0290(10)$ & $0.0297(10)$ & -0.0048 & $0.0033(8)$ & $0.0151(8)$ \\
\hline $\mathrm{C} 26 \mathrm{~A}$ & $0.0391(11)$ & $0.0187(9)$ & $0.0373(11)$ & $-0.0034(8)$ & $-0.0037(9)$ & $0.0084(8)$ \\
\hline C27A & $0.0428(11)$ & $0.0195(9)$ & $0.0413(11)$ & $0.0061(8)$ & $0.0080(9)$ & $-0.0007(8)$ \\
\hline $\mathrm{C} 28 \mathrm{~A}$ & $0.0328(10)$ & $0.0222(9)$ & $0.0267(9)$ & $0.0033(7)$ & $0.0118(8)$ & $0.0031(7)$ \\
\hline O1B & $0.0177(6)$ & $0.0252(6)$ & $0.0191(6)$ & $0.0025(5)$ & $0.0061(5)$ & $0.0050(5)$ \\
\hline $\mathrm{O} 2 \mathrm{~B}$ & $0.0211(6)$ & $0.0377(7)$ & 0.0260 & $0.0010(5)$ & $0.0055(5)$ & $0.0085(5)$ \\
\hline $\mathrm{O} 3 \mathrm{~B}$ & $0.0207(6)$ & $0.0306(7)$ & $0.0213(6)$ & $0.0038(5)$ & $0.0076(5)$ & $0.0055(5)$ \\
\hline O4B & $0.0400(7)$ & $0.0223(6)$ & $0.0211(6)$ & $0.0064(5)$ & -0.0040 & $-0.0007(5)$ \\
\hline O5B & $0.0327(7)$ & $0.0167(6)$ & $0.0159(6)$ & $0.0025(5)$ & $-0.0006(5)$ & $0.0021(4)$ \\
\hline N1B & $0.0146(6)$ & $0.0194(7)$ & $0.0170(6)$ & $0.0017(5)$ & $0.0037(5)$ & $0.0037(5)$ \\
\hline $\mathrm{N} 2 \mathrm{~B}$ & $0.0214(7)$ & $0.0149(6)$ & $0.0178(7)$ & $0.0008(5)$ & $0.0023(5)$ & $0.0015(5)$ \\
\hline N3B & $0.0317(8)$ & $0.0158(7)$ & $0.0142(6)$ & $0.0036(6)$ & $0.0020(6)$ & $0.0027(5)$ \\
\hline $\mathrm{C} 1 \mathrm{~B}$ & $0.0231(8)$ & $0.0144(7)$ & $0.0174(8)$ & $0.0009(6)$ & $0.0025(6)$ & $0.0039(6)$ \\
\hline
\end{tabular}


supporting information

$\begin{array}{lllllll}\text { C2B } & 0.0203(8) & 0.0181(8) & 0.0209(8) & 0.0012(6) & 0.0043(6) & 0.0066(6) \\ \text { C3B } & 0.0209(8) & 0.0146(7) & 0.0183(8) & 0.0039(6) & 0.0042(6) & 0.0053(6) \\ \text { C4B } & 0.0198(8) & 0.0175(8) & 0.0134(7) & 0.0016(6) & 0.0013(6) & 0.0020(6) \\ \text { C5B } & 0.0271(9) & 0.0280(9) & 0.0153(8) & -0.0009(7) & 0.0035(7) & 0.0067(7) \\ \text { C6B } & 0.0389(10) & 0.0266(9) & 0.0248(9) & -0.0067(8) & 0.0020(8) & 0.0122(7) \\ \text { C7B } & 0.0354(10) & 0.0197(9) & 0.0307(10) & 0.0037(7) & -0.0047(8) & 0.0046(7) \\ \text { C8B } & 0.0233(9) & 0.0256(9) & 0.0272(9) & 0.0071(7) & 0.0012(7) & 0.0017(7) \\ \text { C9B } & 0.0188(8) & 0.0233(8) & 0.0211(8) & 0.0011(6) & 0.0025(6) & 0.0048(7) \\ \text { C10B } & 0.0197(8) & 0.0204(8) & 0.0188(8) & 0.0021(6) & 0.0033(6) & 0.0013(6) \\ \text { C11B } & 0.0217(8) & 0.0155(8) & 0.0199(8) & 0.0021(6) & 0.0048(6) & 0.0008(6) \\ \text { C12B } & 0.0227(8) & 0.0175(8) & 0.0210(8) & 0.0007(6) & 0.0038(7) & 0.0034(6) \\ \text { C13B } & 0.0373(10) & 0.0191(9) & 0.0265(9) & 0.0070(7) & 0.0037(8) & 0.0036(7) \\ \text { C14B } & 0.0452(12) & 0.0177(9) & 0.0381(11) & -0.0055(8) & 0.0026(9) & 0.0073(8) \\ \text { C15B } & 0.0324(10) & 0.0252(10) & 0.0423(11) & -0.0084(8) & 0.0029(9) & 0.0073(8) \\ \text { C16B } & 0.0227(9) & 0.0242(9) & 0.0327(10) & -0.0005(7) & 0.0058(7) & 0.0052(7) \\ \text { C17B } & 0.0210(8) & 0.0157(7) & 0.0148(7) & 0.0011(6) & 0.0002(6) & 0.0038(6) \\ \text { C18B } & 0.0287(9) & 0.0204(8) & 0.0179(8) & 0.0038(7) & 0.0068(7) & 0.0042(6) \\ \text { C19B } & 0.0379(16) & 0.0201(13) & 0.0310(15) & 0.0027(11) & 0.0130(13) & 0.0006(11) \\ \text { C19C } & 0.046(5) & 0.026(4) & 0.018(4) & 0.023(4) & -0.006(4) & -0.009(3) \\ \text { C20B } & 0.0292(9) & 0.0179(8) & 0.0325(10) & -0.0006(7) & -0.0019(8) & 0.0025(7) \\ \text { C21B } & 0.0210(8) & 0.0225(8) & 0.0210(8) & 0.0000(7) & 0.0010(7) & 0.0050(7) \\ \text { C22B } & 0.0178(7) & 0.0185(8) & 0.0171(8) & 0.0013(6) & 0.0027(6) & 0.0039(6) \\ \text { C23B } & 0.0223(8) & 0.0156(7) & 0.0159(7) & 0.0023(6) & 0.0027(6) & 0.0040(6) \\ \text { C24B } & 0.0210(8) & 0.0206(8) & 0.0174(8) & 0.0023(6) & 0.0041(6) & 0.0052(6) \\ \text { C25B } & 0.0288(9) & 0.0235(9) & 0.0208(8) & 0.0105(7) & 0.0083(7) & 0.0079(7) \\ \text { C26B } & 0.0400(10) & 0.0190(8) & 0.0296(9) & 0.0040(7) & 0.0124(8) & 0.0101(7) \\ \text { C27B } & 0.0298(9) & 0.0250(9) & 0.0356(10) & -0.0056(7) & 0.0082(8) & 0.0096(8) \\ \text { C28B } & 0.0198(8) & 0.0276(9) & 0.0288(9) & 0.0034(7) & 0.0053(7) & 0.0093(7) \\ \text { O1W } & 0.0209(6) & 0.0200(6) & 0.0262(6) & 0.0006(5) & 0.0060(5) & 0.0003(5)\end{array}$

Geometric parameters $\left(\AA,{ }^{\circ}\right)$

\begin{tabular}{llll}
\hline $\mathrm{O} 1 \mathrm{~A}-\mathrm{C} 2 \mathrm{~A}$ & $1.3777(18)$ & $\mathrm{O} 3 \mathrm{~B}-\mathrm{C} 3 \mathrm{~B}$ & $1.2141(19)$ \\
$\mathrm{O} 1 \mathrm{~A}-\mathrm{N} 1 \mathrm{~A}$ & $1.4332(16)$ & $\mathrm{O} 4 \mathrm{~B}-\mathrm{C} 10 \mathrm{~B}$ & $1.237(2)$ \\
$\mathrm{O} 2 \mathrm{~A}-\mathrm{C} 2 \mathrm{~A}$ & $1.1955(19)$ & $\mathrm{O} 5 \mathrm{~B}-\mathrm{C} 22 \mathrm{~B}$ & $1.2391(19)$ \\
$\mathrm{O} 3 \mathrm{~A}-\mathrm{C} 3 \mathrm{~A}$ & $1.2179(18)$ & $\mathrm{N} 1 \mathrm{~B}-\mathrm{C} 3 \mathrm{~B}$ & $1.358(2)$ \\
$\mathrm{O} 4 \mathrm{~A}-\mathrm{C} 10 \mathrm{~A}$ & $1.2344(19)$ & $\mathrm{N} 1 \mathrm{~B}-\mathrm{C} 4 \mathrm{~B}$ & $1.4677(19)$ \\
$\mathrm{O} 5 \mathrm{~A}-\mathrm{C} 22 \mathrm{~A}$ & $1.2403(18)$ & $\mathrm{N} 2 \mathrm{~B}-\mathrm{C} 10 \mathrm{~B}$ & $1.331(2)$ \\
$\mathrm{N} 1 \mathrm{~A}-\mathrm{C} 3 \mathrm{~A}$ & $1.3534(19)$ & $\mathrm{N} 2 \mathrm{~B}-\mathrm{C} 11 \mathrm{~B}$ & $1.465(2)$ \\
$\mathrm{N} 1 \mathrm{~A}-\mathrm{C} 4 \mathrm{~A}$ & $1.4675(19)$ & $\mathrm{N} 2 \mathrm{~B}-\mathrm{H} 2 \mathrm{BA}$ & 0.8800 \\
$\mathrm{~N} 2 \mathrm{~A}-\mathrm{C} 10 \mathrm{~A}$ & $1.332(2)$ & $\mathrm{N} 3 \mathrm{~B}-\mathrm{C} 22 \mathrm{~B}$ & $1.335(2)$ \\
$\mathrm{N} 2 \mathrm{~A}-\mathrm{C} 11 \mathrm{~A}$ & $1.466(2)$ & $\mathrm{N} 3 \mathrm{~B}-\mathrm{C} 23 \mathrm{~B}$ & $1.462(2)$ \\
$\mathrm{N} 2 \mathrm{~A}-\mathrm{H} 2 \mathrm{AA}$ & 0.8800 & $\mathrm{~N} 3 \mathrm{~B}-\mathrm{H} 3 \mathrm{BA}$ & 0.8800 \\
$\mathrm{~N} 3 \mathrm{~A}-\mathrm{C} 22 \mathrm{~A}$ & $1.329(2)$ & $\mathrm{C} 1 \mathrm{~B}-\mathrm{C} 2 \mathrm{~B}$ & $1.513(2)$ \\
$\mathrm{N} 3 \mathrm{~A}-\mathrm{C} 23 \mathrm{~A}$ & $1.4625(19)$ & $\mathrm{C} 1 \mathrm{~B}-\mathrm{C} 3 \mathrm{~B}$ & $1.524(2)$ \\
$\mathrm{N} 3 \mathrm{~A}-\mathrm{H} 3 \mathrm{AA}$ & 0.8800 & $\mathrm{C} 1 \mathrm{~B}-\mathrm{C} 17 \mathrm{~B}$ & $1.544(2)$ \\
$\mathrm{C} 1 \mathrm{~A}-\mathrm{C} 2 \mathrm{~A}$ & $1.512(2)$ & $\mathrm{C} 1 \mathrm{~B}-\mathrm{H} 1 \mathrm{BA}$ & 1.0000 \\
$\mathrm{C} 1 \mathrm{~A}-\mathrm{C} 3 \mathrm{~A}$ & $1.518(2)$ & $\mathrm{C} 4 \mathrm{~B}-\mathrm{C} 5 \mathrm{~B}$ & $1.537(2)$
\end{tabular}




\begin{tabular}{|c|c|c|c|}
\hline $\mathrm{C} 1 \mathrm{~A}-\mathrm{C} 17 \mathrm{~A}$ & $1.545(2)$ & $\mathrm{C} 4 \mathrm{~B}-\mathrm{C} 10 \mathrm{~B}$ & $1.540(2)$ \\
\hline $\mathrm{C} 1 \mathrm{~A}-\mathrm{H} 1 \mathrm{AA}$ & 1.0000 & $\mathrm{C} 4 \mathrm{~B}-\mathrm{C} 9 \mathrm{~B}$ & $1.548(2)$ \\
\hline $\mathrm{C} 4 \mathrm{~A}-\mathrm{C} 5 \mathrm{~A}$ & $1.537(2)$ & $\mathrm{C} 5 \mathrm{~B}-\mathrm{C} 6 \mathrm{~B}$ & $1.526(2)$ \\
\hline $\mathrm{C} 4 \mathrm{~A}-\mathrm{C} 10 \mathrm{~A}$ & $1.539(2)$ & $\mathrm{C} 5 \mathrm{~B}-\mathrm{H} 5 \mathrm{BA}$ & 0.9900 \\
\hline $\mathrm{C} 4 \mathrm{~A}-\mathrm{C} 9 \mathrm{~A}$ & $1.547(2)$ & $\mathrm{C} 5 \mathrm{~B}-\mathrm{H} 5 \mathrm{BB}$ & 0.9900 \\
\hline $\mathrm{C} 5 \mathrm{~A}-\mathrm{C} 6 \mathrm{~A}$ & $1.530(2)$ & $\mathrm{C} 6 \mathrm{~B}-\mathrm{C} 7 \mathrm{~B}$ & $1.522(3)$ \\
\hline C5A-H5AA & 0.9900 & C6B-H6BA & 0.9900 \\
\hline $\mathrm{C} 5 \mathrm{~A}-\mathrm{H} 5 \mathrm{AB}$ & 0.9900 & $\mathrm{C} 6 \mathrm{~B}-\mathrm{H} 6 \mathrm{BB}$ & 0.9900 \\
\hline $\mathrm{C} 6 \mathrm{~A}-\mathrm{C} 7 \mathrm{~A}$ & $1.525(2)$ & $\mathrm{C} 7 \mathrm{~B}-\mathrm{C} 8 \mathrm{~B}$ & $1.513(3)$ \\
\hline C6A-H6AA & 0.9900 & C7B-H7BA & 0.9900 \\
\hline C6A-H6AB & 0.9900 & $\mathrm{C} 7 \mathrm{~B}-\mathrm{H} 7 \mathrm{BB}$ & 0.9900 \\
\hline $\mathrm{C} 7 \mathrm{~A}-\mathrm{C} 8 \mathrm{~A}$ & $1.522(2)$ & $\mathrm{C} 8 \mathrm{~B}-\mathrm{C} 9 \mathrm{~B}$ & $1.523(2)$ \\
\hline C7A-H7AA & 0.9900 & $\mathrm{C} 8 \mathrm{~B}-\mathrm{H} 8 \mathrm{BA}$ & 0.9900 \\
\hline $\mathrm{C} 7 \mathrm{~A}-\mathrm{H} 7 \mathrm{AB}$ & 0.9900 & $\mathrm{C} 8 \mathrm{~B}-\mathrm{H} 8 \mathrm{BB}$ & 0.9900 \\
\hline $\mathrm{C} 8 \mathrm{~A}-\mathrm{C} 9 \mathrm{~A}$ & $1.528(2)$ & C9B-H9BA & 0.9900 \\
\hline C8A-H8AA & 0.9900 & C9B-H9BB & 0.9900 \\
\hline $\mathrm{C} 8 \mathrm{~A}-\mathrm{H} 8 \mathrm{AB}$ & 0.9900 & $\mathrm{C} 11 \mathrm{~B}-\mathrm{C} 12 \mathrm{~B}$ & $1.521(2)$ \\
\hline C9A-H9AA & 0.9900 & $\mathrm{C} 11 \mathrm{~B}-\mathrm{C} 16 \mathrm{~B}$ & $1.528(2)$ \\
\hline $\mathrm{C} 9 \mathrm{~A}-\mathrm{H} 9 \mathrm{AB}$ & 0.9900 & $\mathrm{C} 11 \mathrm{~B}-\mathrm{H} 11 \mathrm{~B}$ & 1.0000 \\
\hline $\mathrm{C} 11 \mathrm{~A}-\mathrm{C} 12 \mathrm{~A}$ & $1.518(2)$ & $\mathrm{C} 12 \mathrm{~B}-\mathrm{C} 13 \mathrm{~B}$ & $1.529(2)$ \\
\hline $\mathrm{C} 11 \mathrm{~A}-\mathrm{C} 16 \mathrm{~A}$ & $1.528(2)$ & $\mathrm{C} 12 \mathrm{~B}-\mathrm{H} 12 \mathrm{C}$ & 0.9900 \\
\hline $\mathrm{C} 11 \mathrm{~A}-\mathrm{H} 11 \mathrm{~A}$ & 1.0000 & $\mathrm{C} 12 \mathrm{~B}-\mathrm{H} 12 \mathrm{D}$ & 0.9900 \\
\hline $\mathrm{C} 12 \mathrm{~A}-\mathrm{C} 13 \mathrm{~A}$ & $1.525(2)$ & $\mathrm{C} 13 \mathrm{~B}-\mathrm{C} 14 \mathrm{~B}$ & $1.527(3)$ \\
\hline $\mathrm{C} 12 \mathrm{~A}-\mathrm{H} 12 \mathrm{~A}$ & 0.9900 & $\mathrm{C} 13 \mathrm{~B}-\mathrm{H} 13 \mathrm{C}$ & 0.9900 \\
\hline $\mathrm{C} 12 \mathrm{~A}-\mathrm{H} 12 \mathrm{~B}$ & 0.9900 & $\mathrm{C} 13 \mathrm{~B}-\mathrm{H} 13 \mathrm{D}$ & 0.9900 \\
\hline $\mathrm{C} 13 \mathrm{~A}-\mathrm{C} 14 \mathrm{~A}$ & $1.525(2)$ & $\mathrm{C} 14 \mathrm{~B}-\mathrm{C} 15 \mathrm{~B}$ & $1.524(3)$ \\
\hline $\mathrm{C} 13 \mathrm{~A}-\mathrm{H} 13 \mathrm{~A}$ & 0.9900 & $\mathrm{C} 14 \mathrm{~B}-\mathrm{H} 14 \mathrm{C}$ & 0.9900 \\
\hline $\mathrm{C} 13 \mathrm{~A}-\mathrm{H} 13 \mathrm{~B}$ & 0.9900 & $\mathrm{C} 14 \mathrm{~B}-\mathrm{H} 14 \mathrm{D}$ & 0.9900 \\
\hline $\mathrm{C} 14 \mathrm{~A}-\mathrm{C} 15 \mathrm{~A}$ & $1.525(3)$ & $\mathrm{C} 15 \mathrm{~B}-\mathrm{C} 16 \mathrm{~B}$ & $1.527(2)$ \\
\hline $\mathrm{C} 14 \mathrm{~A}-\mathrm{H} 14 \mathrm{~A}$ & 0.9900 & $\mathrm{C} 15 \mathrm{~B}-\mathrm{H} 15 \mathrm{C}$ & 0.9900 \\
\hline $\mathrm{C} 14 \mathrm{~A}-\mathrm{H} 14 \mathrm{~B}$ & 0.9900 & $\mathrm{C} 15 \mathrm{~B}-\mathrm{H} 15 \mathrm{D}$ & 0.9900 \\
\hline $\mathrm{C} 15 \mathrm{~A}-\mathrm{C} 16 \mathrm{~A}$ & $1.530(2)$ & $\mathrm{C} 16 \mathrm{~B}-\mathrm{H} 16 \mathrm{C}$ & 0.9900 \\
\hline $\mathrm{C} 15 \mathrm{~A}-\mathrm{H} 15 \mathrm{~A}$ & 0.9900 & $\mathrm{C} 16 \mathrm{~B}-\mathrm{H} 16 \mathrm{D}$ & 0.9900 \\
\hline $\mathrm{C} 15 \mathrm{~A}-\mathrm{H} 15 \mathrm{~B}$ & 0.9900 & $\mathrm{C} 17 \mathrm{~B}-\mathrm{C} 22 \mathrm{~B}$ & $1.530(2)$ \\
\hline $\mathrm{C} 16 \mathrm{~A}-\mathrm{H} 16 \mathrm{~A}$ & 0.9900 & $\mathrm{C} 17 \mathrm{~B}-\mathrm{C} 18 \mathrm{~B}$ & $1.548(2)$ \\
\hline $\mathrm{C} 16 \mathrm{~A}-\mathrm{H} 16 \mathrm{~B}$ & 0.9900 & $\mathrm{C} 17 \mathrm{~B}-\mathrm{C} 21 \mathrm{~B}$ & $1.552(2)$ \\
\hline $\mathrm{C} 17 \mathrm{~A}-\mathrm{C} 22 \mathrm{~A}$ & $1.530(2)$ & $\mathrm{C} 18 \mathrm{~B}-\mathrm{C} 19 \mathrm{C}$ & $1.506(8)$ \\
\hline $\mathrm{C} 17 \mathrm{~A}-\mathrm{C} 18 \mathrm{~A}$ & $1.546(2)$ & $\mathrm{C} 18 \mathrm{~B}-\mathrm{C} 19 \mathrm{~B}$ & $1.528(3)$ \\
\hline $\mathrm{C} 17 \mathrm{~A}-\mathrm{C} 21 \mathrm{~A}$ & $1.548(2)$ & $\mathrm{C} 18 \mathrm{~B}-\mathrm{H} 18 \mathrm{C}$ & 0.9600 \\
\hline $\mathrm{C} 18 \mathrm{~A}-\mathrm{C} 19 \mathrm{~A}$ & $1.534(2)$ & $\mathrm{C} 18 \mathrm{~B}-\mathrm{H} 18 \mathrm{D}$ & 0.9602 \\
\hline $\mathrm{C} 18 \mathrm{~A}-\mathrm{H} 18 \mathrm{~A}$ & 0.9900 & $\mathrm{C} 19 \mathrm{~B}-\mathrm{C} 20 \mathrm{~B}$ & $1.516(3)$ \\
\hline $\mathrm{C} 18 \mathrm{~A}-\mathrm{H} 18 \mathrm{~B}$ & 0.9900 & $\mathrm{C} 19 \mathrm{~B}-\mathrm{H} 19 \mathrm{C}$ & 0.9900 \\
\hline $\mathrm{C} 19 \mathrm{~A}-\mathrm{C} 20 \mathrm{~A}$ & $1.543(2)$ & $\mathrm{C} 19 \mathrm{~B}-\mathrm{H} 19 \mathrm{D}$ & 0.9900 \\
\hline C19A-H19A & 0.9900 & $\mathrm{C} 19 \mathrm{C}-\mathrm{C} 20 \mathrm{~B}$ & $1.653(10)$ \\
\hline $\mathrm{C} 19 \mathrm{~A}-\mathrm{H} 19 \mathrm{~B}$ & 0.9900 & $\mathrm{C} 19 \mathrm{C}-\mathrm{H} 19 \mathrm{E}$ & 0.9900 \\
\hline $\mathrm{C} 20 \mathrm{~A}-\mathrm{C} 21 \mathrm{~A}$ & $1.527(2)$ & $\mathrm{C} 19 \mathrm{C}-\mathrm{H} 19 \mathrm{~F}$ & 0.9900 \\
\hline $\mathrm{C} 20 \mathrm{~A}-\mathrm{H} 20 \mathrm{~A}$ & 0.9900 & $\mathrm{C} 20 \mathrm{~B}-\mathrm{C} 21 \mathrm{~B}$ & $1.522(2)$ \\
\hline $\mathrm{C} 20 \mathrm{~A}-\mathrm{H} 20 \mathrm{~B}$ & 0.9900 & $\mathrm{C} 20 \mathrm{~B}-\mathrm{H} 20 \mathrm{C}$ & 0.9600 \\
\hline
\end{tabular}




\begin{tabular}{|c|c|c|c|}
\hline $\mathrm{C} 21 \mathrm{~A}-\mathrm{H} 21 \mathrm{~A}$ & 0.9900 & $\mathrm{C} 20 \mathrm{~B}-\mathrm{H} 20 \mathrm{D}$ & 0.9598 \\
\hline $\mathrm{C} 21 \mathrm{~A}-\mathrm{H} 21 \mathrm{~B}$ & 0.9900 & $\mathrm{C} 21 \mathrm{~B}-\mathrm{H} 21 \mathrm{C}$ & 0.9900 \\
\hline $\mathrm{C} 23 \mathrm{~A}-\mathrm{C} 28 \mathrm{~A}$ & $1.523(2)$ & $\mathrm{C} 21 \mathrm{~B}-\mathrm{H} 21 \mathrm{D}$ & 0.9900 \\
\hline $\mathrm{C} 23 \mathrm{~A}-\mathrm{C} 24 \mathrm{~A}$ & $1.527(2)$ & $\mathrm{C} 23 \mathrm{~B}-\mathrm{C} 24 \mathrm{~B}$ & $1.524(2)$ \\
\hline $\mathrm{C} 23 \mathrm{~A}-\mathrm{H} 23 \mathrm{~A}$ & 1.0000 & $\mathrm{C} 23 \mathrm{~B}-\mathrm{C} 28 \mathrm{~B}$ & $1.526(2)$ \\
\hline $\mathrm{C} 24 \mathrm{~A}-\mathrm{C} 25 \mathrm{~A}$ & $1.526(2)$ & $\mathrm{C} 23 \mathrm{~B}-\mathrm{H} 23 \mathrm{~B}$ & 1.0000 \\
\hline $\mathrm{C} 24 \mathrm{~A}-\mathrm{H} 24 \mathrm{~B}$ & 0.9900 & $\mathrm{C} 24 \mathrm{~B}-\mathrm{C} 25 \mathrm{~B}$ & $1.528(2)$ \\
\hline $\mathrm{C} 24 \mathrm{~A}-\mathrm{H} 24 \mathrm{C}$ & 0.9900 & $\mathrm{C} 24 \mathrm{~B}-\mathrm{H} 24 \mathrm{~A}$ & 0.9900 \\
\hline $\mathrm{C} 25 \mathrm{~A}-\mathrm{C} 26 \mathrm{~A}$ & $1.522(3)$ & $\mathrm{C} 24 \mathrm{~B}-\mathrm{H} 24 \mathrm{D}$ & 0.9900 \\
\hline $\mathrm{C} 25 \mathrm{~A}-\mathrm{H} 25 \mathrm{~A}$ & 0.9900 & $\mathrm{C} 25 \mathrm{~B}-\mathrm{C} 26 \mathrm{~B}$ & $1.523(2)$ \\
\hline $\mathrm{C} 25 \mathrm{~A}-\mathrm{H} 25 \mathrm{~B}$ & 0.9900 & $\mathrm{C} 25 \mathrm{~B}-\mathrm{H} 25 \mathrm{C}$ & 0.9900 \\
\hline $\mathrm{C} 26 \mathrm{~A}-\mathrm{C} 27 \mathrm{~A}$ & $1.520(3)$ & $\mathrm{C} 25 \mathrm{~B}-\mathrm{H} 25 \mathrm{D}$ & 0.9900 \\
\hline $\mathrm{C} 26 \mathrm{~A}-\mathrm{H} 26 \mathrm{~A}$ & 0.9900 & $\mathrm{C} 26 \mathrm{~B}-\mathrm{C} 27 \mathrm{~B}$ & $1.523(3)$ \\
\hline $\mathrm{C} 26 \mathrm{~A}-\mathrm{H} 26 \mathrm{~B}$ & 0.9900 & $\mathrm{C} 26 \mathrm{~B}-\mathrm{H} 26 \mathrm{C}$ & 0.9900 \\
\hline $\mathrm{C} 27 \mathrm{~A}-\mathrm{C} 28 \mathrm{~A}$ & $1.528(2)$ & $\mathrm{C} 26 \mathrm{~B}-\mathrm{H} 26 \mathrm{D}$ & 0.9900 \\
\hline $\mathrm{C} 27 \mathrm{~A}-\mathrm{H} 27 \mathrm{~A}$ & 0.9900 & $\mathrm{C} 27 \mathrm{~B}-\mathrm{C} 28 \mathrm{~B}$ & $1.528(2)$ \\
\hline $\mathrm{C} 27 \mathrm{~A}-\mathrm{H} 27 \mathrm{~B}$ & 0.9900 & $\mathrm{C} 27 \mathrm{~B}-\mathrm{H} 27 \mathrm{C}$ & 0.9900 \\
\hline $\mathrm{C} 28 \mathrm{~A}-\mathrm{H} 28 \mathrm{~A}$ & 0.9900 & $\mathrm{C} 27 \mathrm{~B}-\mathrm{H} 27 \mathrm{D}$ & 0.9900 \\
\hline $\mathrm{C} 28 \mathrm{~A}-\mathrm{H} 28 \mathrm{~B}$ & 0.9900 & $\mathrm{C} 28 \mathrm{~B}-\mathrm{H} 28 \mathrm{C}$ & 0.9900 \\
\hline $\mathrm{O} 1 \mathrm{~B}-\mathrm{C} 2 \mathrm{~B}$ & $1.3633(19)$ & $\mathrm{C} 28 \mathrm{~B}-\mathrm{H} 28 \mathrm{D}$ & 0.9900 \\
\hline $\mathrm{O} 1 \mathrm{~B}-\mathrm{N} 1 \mathrm{~B}$ & $1.4415(16)$ & $\mathrm{O} 1 \mathrm{~W}-\mathrm{H} 1 \mathrm{WA}$ & $0.86(3)$ \\
\hline $\mathrm{O} 2 \mathrm{~B}-\mathrm{C} 2 \mathrm{~B}$ & $1.200(2)$ & $\mathrm{O} 1 \mathrm{~W}-\mathrm{H} 1 \mathrm{WB}$ & $0.89(3)$ \\
\hline $\mathrm{C} 2 \mathrm{~A}-\mathrm{O} 1 \mathrm{~A}-\mathrm{N} 1 \mathrm{~A}$ & $107.90(11)$ & $\mathrm{C} 22 \mathrm{~B}-\mathrm{N} 3 \mathrm{~B}-\mathrm{H} 3 \mathrm{BA}$ & 118.6 \\
\hline $\mathrm{C} 3 \mathrm{~A}-\mathrm{N} 1 \mathrm{~A}-\mathrm{O} 1 \mathrm{~A}$ & $111.76(12)$ & $\mathrm{C} 23 \mathrm{~B}-\mathrm{N} 3 \mathrm{~B}-\mathrm{H} 3 \mathrm{BA}$ & 118.6 \\
\hline $\mathrm{C} 3 \mathrm{~A}-\mathrm{N} 1 \mathrm{~A}-\mathrm{C} 4 \mathrm{~A}$ & $135.16(13)$ & $\mathrm{C} 2 \mathrm{~B}-\mathrm{C} 1 \mathrm{~B}-\mathrm{C} 3 \mathrm{~B}$ & $102.17(12)$ \\
\hline $\mathrm{O} 1 \mathrm{~A}-\mathrm{N} 1 \mathrm{~A}-\mathrm{C} 4 \mathrm{~A}$ & $112.43(11)$ & $\mathrm{C} 2 \mathrm{~B}-\mathrm{C} 1 \mathrm{~B}-\mathrm{C} 17 \mathrm{~B}$ & $115.77(13)$ \\
\hline $\mathrm{C} 10 \mathrm{~A}-\mathrm{N} 2 \mathrm{~A}-\mathrm{C} 11 \mathrm{~A}$ & $120.88(13)$ & $\mathrm{C} 3 \mathrm{~B}-\mathrm{C} 1 \mathrm{~B}-\mathrm{C} 17 \mathrm{~B}$ & $115.01(13)$ \\
\hline $\mathrm{C} 10 \mathrm{~A}-\mathrm{N} 2 \mathrm{~A}-\mathrm{H} 2 \mathrm{AA}$ & 119.6 & $\mathrm{C} 2 \mathrm{~B}-\mathrm{C} 1 \mathrm{~B}-\mathrm{H} 1 \mathrm{BA}$ & 107.8 \\
\hline $\mathrm{C} 11 \mathrm{~A}-\mathrm{N} 2 \mathrm{~A}-\mathrm{H} 2 \mathrm{AA}$ & 119.6 & $\mathrm{C} 3 \mathrm{~B}-\mathrm{C} 1 \mathrm{~B}-\mathrm{H} 1 \mathrm{BA}$ & 107.8 \\
\hline $\mathrm{C} 22 \mathrm{~A}-\mathrm{N} 3 \mathrm{~A}-\mathrm{C} 23 \mathrm{~A}$ & $123.23(13)$ & $\mathrm{C} 17 \mathrm{~B}-\mathrm{C} 1 \mathrm{~B}-\mathrm{H} 1 \mathrm{BA}$ & 107.8 \\
\hline $\mathrm{C} 22 \mathrm{~A}-\mathrm{N} 3 \mathrm{~A}-\mathrm{H} 3 \mathrm{AA}$ & 118.4 & $\mathrm{O} 2 \mathrm{~B}-\mathrm{C} 2 \mathrm{~B}-\mathrm{O} 1 \mathrm{~B}$ & $119.02(15)$ \\
\hline $\mathrm{C} 23 \mathrm{~A}-\mathrm{N} 3 \mathrm{~A}-\mathrm{H} 3 \mathrm{AA}$ & 118.4 & $\mathrm{O} 2 \mathrm{~B}-\mathrm{C} 2 \mathrm{~B}-\mathrm{C} 1 \mathrm{~B}$ & $131.38(15)$ \\
\hline $\mathrm{C} 2 \mathrm{~A}-\mathrm{C} 1 \mathrm{~A}-\mathrm{C} 3 \mathrm{~A}$ & $102.38(12)$ & $\mathrm{O} 1 \mathrm{~B}-\mathrm{C} 2 \mathrm{~B}-\mathrm{C} 1 \mathrm{~B}$ & $109.47(13)$ \\
\hline $\mathrm{C} 2 \mathrm{~A}-\mathrm{C} 1 \mathrm{~A}-\mathrm{C} 17 \mathrm{~A}$ & $115.89(12)$ & $\mathrm{O} 3 \mathrm{~B}-\mathrm{C} 3 \mathrm{~B}-\mathrm{N} 1 \mathrm{~B}$ & $125.39(15)$ \\
\hline $\mathrm{C} 3 \mathrm{~A}-\mathrm{C} 1 \mathrm{~A}-\mathrm{C} 17 \mathrm{~A}$ & $115.38(12)$ & $\mathrm{O} 3 \mathrm{~B}-\mathrm{C} 3 \mathrm{~B}-\mathrm{C} 1 \mathrm{~B}$ & $127.42(14)$ \\
\hline $\mathrm{C} 2 \mathrm{~A}-\mathrm{C} 1 \mathrm{~A}-\mathrm{H} 1 \mathrm{AA}$ & 107.6 & $\mathrm{~N} 1 \mathrm{~B}-\mathrm{C} 3 \mathrm{~B}-\mathrm{C} 1 \mathrm{~B}$ & $107.13(13)$ \\
\hline $\mathrm{C} 3 \mathrm{~A}-\mathrm{C} 1 \mathrm{~A}-\mathrm{H} 1 \mathrm{AA}$ & 107.6 & $\mathrm{~N} 1 \mathrm{~B}-\mathrm{C} 4 \mathrm{~B}-\mathrm{C} 5 \mathrm{~B}$ & $109.19(13)$ \\
\hline $\mathrm{C} 17 \mathrm{~A}-\mathrm{C} 1 \mathrm{~A}-\mathrm{H} 1 \mathrm{AA}$ & 107.6 & $\mathrm{~N} 1 \mathrm{~B}-\mathrm{C} 4 \mathrm{~B}-\mathrm{C} 10 \mathrm{~B}$ & $112.50(12)$ \\
\hline $\mathrm{O} 2 \mathrm{~A}-\mathrm{C} 2 \mathrm{~A}-\mathrm{O} 1 \mathrm{~A}$ & $118.70(14)$ & $\mathrm{C} 5 \mathrm{~B}-\mathrm{C} 4 \mathrm{~B}-\mathrm{C} 10 \mathrm{~B}$ & $109.95(13)$ \\
\hline $\mathrm{O} 2 \mathrm{~A}-\mathrm{C} 2 \mathrm{~A}-\mathrm{C} 1 \mathrm{~A}$ & $132.03(14)$ & $\mathrm{N} 1 \mathrm{~B}-\mathrm{C} 4 \mathrm{~B}-\mathrm{C} 9 \mathrm{~B}$ & $109.71(12)$ \\
\hline $\mathrm{O} 1 \mathrm{~A}-\mathrm{C} 2 \mathrm{~A}-\mathrm{C} 1 \mathrm{~A}$ & $109.15(12)$ & $\mathrm{C} 5 \mathrm{~B}-\mathrm{C} 4 \mathrm{~B}-\mathrm{C} 9 \mathrm{~B}$ & $109.15(13)$ \\
\hline $\mathrm{O} 3 \mathrm{~A}-\mathrm{C} 3 \mathrm{~A}-\mathrm{N} 1 \mathrm{~A}$ & $124.94(14)$ & $\mathrm{C} 10 \mathrm{~B}-\mathrm{C} 4 \mathrm{~B}-\mathrm{C} 9 \mathrm{~B}$ & $106.27(13)$ \\
\hline $\mathrm{O} 3 \mathrm{~A}-\mathrm{C} 3 \mathrm{~A}-\mathrm{C} 1 \mathrm{~A}$ & $127.48(14)$ & $\mathrm{C} 6 \mathrm{~B}-\mathrm{C} 5 \mathrm{~B}-\mathrm{C} 4 \mathrm{~B}$ & $112.34(14)$ \\
\hline $\mathrm{N} 1 \mathrm{~A}-\mathrm{C} 3 \mathrm{~A}-\mathrm{C} 1 \mathrm{~A}$ & $107.48(13)$ & $\mathrm{C} 6 \mathrm{~B}-\mathrm{C} 5 \mathrm{~B}-\mathrm{H} 5 \mathrm{BA}$ & 109.1 \\
\hline $\mathrm{N} 1 \mathrm{~A}-\mathrm{C} 4 \mathrm{~A}-\mathrm{C} 5 \mathrm{~A}$ & $109.09(12)$ & $\mathrm{C} 4 \mathrm{~B}-\mathrm{C} 5 \mathrm{~B}-\mathrm{H} 5 \mathrm{BA}$ & 109.1 \\
\hline $\mathrm{N} 1 \mathrm{~A}-\mathrm{C} 4 \mathrm{~A}-\mathrm{C} 10 \mathrm{~A}$ & $111.89(12)$ & $\mathrm{C} 6 \mathrm{~B}-\mathrm{C} 5 \mathrm{~B}-\mathrm{H} 5 \mathrm{BB}$ & 109.1 \\
\hline $\mathrm{C} 5 \mathrm{~A}-\mathrm{C} 4 \mathrm{~A}-\mathrm{C} 10 \mathrm{~A}$ & $109.56(12)$ & $\mathrm{C} 4 \mathrm{~B}-\mathrm{C} 5 \mathrm{~B}-\mathrm{H} 5 \mathrm{BB}$ & 109.1 \\
\hline
\end{tabular}




$\begin{array}{ll}\text { N1A-C4A-C9A } & 109.53(12) \\ \text { C5A-C4A-C9A } & 108.68(13) \\ \text { C10A-C4A-C9A } & 108.04(12) \\ \text { C6A-C5A-C4A } & 111.94(13) \\ \text { C6A-C5A-H5AA } & 109.2 \\ \text { C4A-C5A-H5AA } & 109.2 \\ \text { C6A-C5A-H5AB } & 109.2 \\ \text { C4A-C5A-H5AB } & 109.2 \\ \text { H5AA-C5A-H5AB } & 107.9 \\ \text { C7A-C6A-C5A } & 111.67(14) \\ \text { C7A-C6A-H6AA } & 109.3 \\ \text { C5A-C6A-H6AA } & 109.3 \\ \text { C7A-C6A-H6AB } & 109.3 \\ \text { C5A-C6A-H6AB } & 109.3 \\ \text { H6AA-C6A-H6AB } & 107.9 \\ \text { C8A-C7A-C6A } & 110.95(13) \\ \text { C8A-C7A-H7AA } & 109.4 \\ \text { C6A-C7A-H7AA } & 109.4 \\ \text { C8A-C7A-H7AB } & 109.4 \\ \text { C6A-C7A-H7AB } & 109.4 \\ \text { H7AA-C7A-H7AB } & 108.0 \\ \text { C7A-C8A-C9A } & 111.98(13) \\ \text { C7A-C8A-H8AA } & 109.2 \\ \text { C9A-C8A-H8AA } & 109.2 \\ \text { C7A-C8A-H8AB } & 109.2 \\ \text { C9A-C8A-H8AB } & 109.2 \\ \text { H8AA-C8A-H8AB } & 107.9 \\ \text { C8A-C9A-C4A } & 112.35(13) \\ \text { C8A-C9A-H9AA } & 109.1 \\ \text { C4A-C9A-H9AA } & 109.1 \\ \text { C8A-C9A-H9AB } & 109.1 \\ \text { C4A-C9A-H9AB } & 109.1 \\ \text { H9AA-C9A-H9AB } & 107.9 \\ \text { O4A-C10A-N2A } & 122.80(15) \\ \text { O4A-C10A-C4A } & 118.48(14) \\ \text { N2A-C10A-C4A } & 118.70(13) \\ \text { N2A-C11A-C12A } & 109.72(13) \\ \text { N2A-C11A-C16A } & 111.55(13) \\ \text { C12A-C11A-C16A } & 110.71(14) \\ \text { N2A-C11A-H11A } & 108.3 \\ \text { C12A-C11A-H11A } & 108.3 \\ \text { C16A-C11A-H11A } & 108.3 \\ \text { C11A-C12A-C13A } & 111.27(14) \\ \text { C11A-C12A-H12A } & 109.4 \\ \text { C13A-C12A-H12A } & 109.4 \\ \text { C11A-C12A-H12B } & 109.4 \\ \text { C13A-C12A-H12B } & 109.4 \\ \text { H12A-C12A-H12B } & \\ & \end{array}$

\begin{tabular}{|c|c|}
\hline $\mathrm{H} 5 \mathrm{BA}-\mathrm{C} 5 \mathrm{~B}-\mathrm{H} 5 \mathrm{BB}$ & 107.9 \\
\hline $\mathrm{C} 7 \mathrm{~B}-\mathrm{C} 6 \mathrm{~B}-\mathrm{C} 5 \mathrm{~B}$ & $111.64(14)$ \\
\hline $\mathrm{C} 7 \mathrm{~B}-\mathrm{C} 6 \mathrm{~B}-\mathrm{H} 6 \mathrm{BA}$ & 109.3 \\
\hline $\mathrm{C} 5 \mathrm{~B}-\mathrm{C} 6 \mathrm{~B}-\mathrm{H} 6 \mathrm{BA}$ & 109.3 \\
\hline $\mathrm{C} 7 \mathrm{~B}-\mathrm{C} 6 \mathrm{~B}-\mathrm{H} 6 \mathrm{BB}$ & 109.3 \\
\hline $\mathrm{C} 5 \mathrm{~B}-\mathrm{C} 6 \mathrm{~B}-\mathrm{H} 6 \mathrm{BB}$ & 109.3 \\
\hline $\mathrm{H} 6 \mathrm{BA}-\mathrm{C} 6 \mathrm{~B}-\mathrm{H} 6 \mathrm{BB}$ & 108.0 \\
\hline $\mathrm{C} 8 \mathrm{~B}-\mathrm{C} 7 \mathrm{~B}-\mathrm{C} 6 \mathrm{~B}$ & $110.84(14)$ \\
\hline $\mathrm{C} 8 \mathrm{~B}-\mathrm{C} 7 \mathrm{~B}-\mathrm{H} 7 \mathrm{BA}$ & 109.5 \\
\hline $\mathrm{C} 6 \mathrm{~B}-\mathrm{C} 7 \mathrm{~B}-\mathrm{H} 7 \mathrm{BA}$ & 109.5 \\
\hline $\mathrm{C} 8 \mathrm{~B}-\mathrm{C} 7 \mathrm{~B}-\mathrm{H} 7 \mathrm{BB}$ & 109.5 \\
\hline $\mathrm{C} 6 \mathrm{~B}-\mathrm{C} 7 \mathrm{~B}-\mathrm{H} 7 \mathrm{BB}$ & 109.5 \\
\hline $\mathrm{H} 7 \mathrm{BA}-\mathrm{C} 7 \mathrm{~B}-\mathrm{H} 7 \mathrm{BB}$ & 108.1 \\
\hline $\mathrm{C} 7 \mathrm{~B}-\mathrm{C} 8 \mathrm{~B}-\mathrm{C} 9 \mathrm{~B}$ & $111.20(14)$ \\
\hline $\mathrm{C} 7 \mathrm{~B}-\mathrm{C} 8 \mathrm{~B}-\mathrm{H} 8 \mathrm{BA}$ & 109.4 \\
\hline $\mathrm{C} 9 \mathrm{~B}-\mathrm{C} 8 \mathrm{~B}-\mathrm{H} 8 \mathrm{BA}$ & 109.4 \\
\hline $\mathrm{C} 7 \mathrm{~B}-\mathrm{C} 8 \mathrm{~B}-\mathrm{H} 8 \mathrm{BB}$ & 109.4 \\
\hline $\mathrm{C} 9 \mathrm{~B}-\mathrm{C} 8 \mathrm{~B}-\mathrm{H} 8 \mathrm{BB}$ & 109.4 \\
\hline $\mathrm{H} 8 \mathrm{BA}-\mathrm{C} 8 \mathrm{~B}-\mathrm{H} 8 \mathrm{BB}$ & 108.0 \\
\hline $\mathrm{C} 8 \mathrm{~B}-\mathrm{C} 9 \mathrm{~B}-\mathrm{C} 4 \mathrm{~B}$ & $113.51(14)$ \\
\hline $\mathrm{C} 8 \mathrm{~B}-\mathrm{C} 9 \mathrm{~B}-\mathrm{H} 9 \mathrm{BA}$ & 108.9 \\
\hline $\mathrm{C} 4 \mathrm{~B}-\mathrm{C} 9 \mathrm{~B}-\mathrm{H} 9 \mathrm{BA}$ & 108.9 \\
\hline $\mathrm{C} 8 \mathrm{~B}-\mathrm{C} 9 \mathrm{~B}-\mathrm{H} 9 \mathrm{BB}$ & 108.9 \\
\hline $\mathrm{C} 4 \mathrm{~B}-\mathrm{C} 9 \mathrm{~B}-\mathrm{H} 9 \mathrm{BB}$ & 108.9 \\
\hline $\mathrm{H} 9 \mathrm{BA}-\mathrm{C} 9 \mathrm{~B}-\mathrm{H} 9 \mathrm{BB}$ & 107.7 \\
\hline $\mathrm{O} 4 \mathrm{~B}-\mathrm{C} 10 \mathrm{~B}-\mathrm{N} 2 \mathrm{~B}$ & $123.39(15)$ \\
\hline $\mathrm{O} 4 \mathrm{~B}-\mathrm{C} 10 \mathrm{~B}-\mathrm{C} 4 \mathrm{~B}$ & $117.72(14)$ \\
\hline $\mathrm{N} 2 \mathrm{~B}-\mathrm{C} 10 \mathrm{~B}-\mathrm{C} 4 \mathrm{~B}$ & $118.74(14)$ \\
\hline $\mathrm{N} 2 \mathrm{~B}-\mathrm{C} 11 \mathrm{~B}-\mathrm{C} 12 \mathrm{~B}$ & $110.02(13)$ \\
\hline $\mathrm{N} 2 \mathrm{~B}-\mathrm{C} 11 \mathrm{~B}-\mathrm{C} 16 \mathrm{~B}$ & $111.03(13)$ \\
\hline $\mathrm{C} 12 \mathrm{~B}-\mathrm{C} 11 \mathrm{~B}-\mathrm{C} 16 \mathrm{~B}$ & $110.74(14)$ \\
\hline $\mathrm{N} 2 \mathrm{~B}-\mathrm{C} 11 \mathrm{~B}-\mathrm{H} 11 \mathrm{~B}$ & 108.3 \\
\hline $\mathrm{C} 12 \mathrm{~B}-\mathrm{C} 11 \mathrm{~B}-\mathrm{H} 11 \mathrm{~B}$ & 108.3 \\
\hline $\mathrm{C} 16 \mathrm{~B}-\mathrm{C} 11 \mathrm{~B}-\mathrm{H} 11 \mathrm{~B}$ & 108.3 \\
\hline $\mathrm{C} 11 \mathrm{~B}-\mathrm{C} 12 \mathrm{~B}-\mathrm{C} 13 \mathrm{~B}$ & $110.40(13)$ \\
\hline $\mathrm{C} 11 \mathrm{~B}-\mathrm{C} 12 \mathrm{~B}-\mathrm{H} 12 \mathrm{C}$ & 109.6 \\
\hline $\mathrm{C} 13 \mathrm{~B}-\mathrm{C} 12 \mathrm{~B}-\mathrm{H} 12 \mathrm{C}$ & 109.6 \\
\hline $\mathrm{C} 11 \mathrm{~B}-\mathrm{C} 12 \mathrm{~B}-\mathrm{H} 12 \mathrm{D}$ & 109.6 \\
\hline $\mathrm{C} 13 \mathrm{~B}-\mathrm{C} 12 \mathrm{~B}-\mathrm{H} 12 \mathrm{D}$ & 109.6 \\
\hline $\mathrm{H} 12 \mathrm{C}-\mathrm{C} 12 \mathrm{~B}-\mathrm{H} 12 \mathrm{D}$ & 108.1 \\
\hline $\mathrm{C} 14 \mathrm{~B}-\mathrm{C} 13 \mathrm{~B}-\mathrm{C} 12 \mathrm{~B}$ & $110.15(15)$ \\
\hline $\mathrm{C} 14 \mathrm{~B}-\mathrm{C} 13 \mathrm{~B}-\mathrm{H} 13 \mathrm{C}$ & 109.6 \\
\hline $\mathrm{C} 12 \mathrm{~B}-\mathrm{C} 13 \mathrm{~B}-\mathrm{H} 13 \mathrm{C}$ & 109.6 \\
\hline $\mathrm{C} 14 \mathrm{~B}-\mathrm{C} 13 \mathrm{~B}-\mathrm{H} 13 \mathrm{D}$ & 109.6 \\
\hline $\mathrm{C} 12 \mathrm{~B}-\mathrm{C} 13 \mathrm{~B}-\mathrm{H} 13 \mathrm{D}$ & 109.6 \\
\hline $\mathrm{H} 13 \mathrm{C}-\mathrm{C} 13 \mathrm{~B}-\mathrm{H} 13 \mathrm{D}$ & 108.1 \\
\hline $\mathrm{C} 15 \mathrm{~B}-\mathrm{C} 14 \mathrm{~B}-\mathrm{C} 13 \mathrm{~B}$ & $110.93(15)$ \\
\hline $\mathrm{C} 15 \mathrm{~B}-\mathrm{C} 14 \mathrm{~B}-\mathrm{H} 14 \mathrm{C}$ & 109.5 \\
\hline
\end{tabular}




$\begin{array}{ll}\text { C14A-C13A-C12A } & 111.03(14) \\ \text { C14A-C13A-H13A } & 109.4 \\ \text { C12A-C13A-H13A } & 109.4 \\ \text { C14A-C13A-H13B } & 109.4 \\ \text { C12A-C13A-H13B } & 109.4 \\ \text { H13A-C13A-H13B } & 108.0 \\ \text { C13A-C14A-C15A } & 110.85(15) \\ \text { C13A-C14A-H14A } & 109.5 \\ \text { C15A-C14A-H14A } & 109.5 \\ \text { C13A-C14A-H14B } & 109.5 \\ \text { C15A-C14A-H14B } & 109.5 \\ \text { H14A-C14A-H14B } & 108.1 \\ \text { C14A-C15A-C16A } & 111.26(15) \\ \text { C14A-C15A-H15A } & 109.4 \\ \text { C16A-C15A-H15A } & 109.4 \\ \text { C14A-C15A-H15B } & 109.4 \\ \text { C16A-C15A-H15B } & 109.4 \\ \text { H15A-C15A-H15B } & 108.0 \\ \text { C11A-C16A-C15A } & 110.48(14) \\ \text { C11A-C16A-H16A } & 109.6 \\ \text { C15A-C16A-H16A } & 109.6 \\ \text { C11A-C16A-H16B } & 109.6 \\ \text { C15A-C16A-H16B } & 109.6 \\ \text { H16A-C16A-H16B } & 108.1 \\ \text { C22A-C17A-C1A } & 106.74(12) \\ \text { C22A-C17A-C18A } & 115.76(13) \\ \text { C1A-C17A-C18A } & 110.46(12) \\ \text { C22A-C17A-C21A } & 112.27(12) \\ \text { C1A-C17A-C21A } & 110.58(12) \\ \text { C18A-C17A-C21A } & 101.02(12) \\ \text { C19A-C18A-C17A } & 105.19(13) \\ \text { C19A-C18A-H18A } & 110.7 \\ \text { C17A-C18A-H18A } & 110.7 \\ \text { C19A-C18A-H18B } & 110.7 \\ \text { C17A-C18A-H18B } & 110.7 \\ \text { H18A-C18A-H18B } & 108.8 \\ \text { C18A-C19A-C20A } & 106.64(14) \\ \text { C18A-C19A-H19A } & 110.4 \\ \text { C20A-C19A-H19A } & 110.4 \\ \text { C18A-C19A-H19B } & 110.4 \\ \text { C20A-C19A-H19B } & 110.4 \\ \text { H19A-C19A-H19B } & 108.6 \\ \text { C21A-C20A-C19A } & 104.93(13) \\ \text { C21A-C20A-H20A } & 110.8 \\ \text { C19A-C20A-H20A } & 110.8 \\ \text { C21A-C20A-H20B } & 110.8 \\ \text { C19A-C20A-H20B } & 110.8 \\ \text { H20A-C20A-H20B } & 108.8 \\ & \end{array}$

$\begin{array}{ll}\text { C13B-C14B-H14C } & 109.5 \\ \text { C15B-C14B-H14D } & 109.5 \\ \text { C13B-C14B-H14D } & 109.5 \\ \text { H14C-C14B-H14D } & 108.0 \\ \text { C14B-C15B-C16B } & 110.77(15) \\ \text { C14B-C15B-H15C } & 109.5 \\ \text { C16B-C15B-H15C } & 109.5 \\ \text { C14B-C15B-H15D } & 109.5 \\ \text { C16B-C15B-H15D } & 109.5 \\ \text { H15C-C15B-H15D } & 108.1 \\ \text { C15B-C16B-C11B } & 111.12(14) \\ \text { C15B-C16B-H16C } & 109.4 \\ \text { C11B-C16B-H16C } & 109.4 \\ \text { C15B-C16B-H16D } & 109.4 \\ \text { C11B-C16B-H16D } & 109.4 \\ \text { H16C-C16B-H16D } & 108.0 \\ \text { C22B-C17B-C1B } & 106.34(12) \\ \text { C22B-C17B-C18B } & 114.76(13) \\ \text { C1B-C17B-C18B } & 110.93(13) \\ \text { C22B-C17B-C21B } & 112.20(13) \\ \text { C1B-C17B-C21B } & 110.94(13) \\ \text { C18B-C17B-C21B } & 101.75(13) \\ \text { C19C-C18B-C19B } & 30.1(3) \\ \text { C19C-C18B-C17B } & 109.7(4) \\ \text { C19B-C18B-C17B } & 104.39(15) \\ \text { C19C-C18B-H18C } & 130.2 \\ \text { C19B-C18B-H18C } & 111.0 \\ \text { C17B-C18B-H18C } & 111.0 \\ \text { C19C-C18B-H18D } & 81.5 \\ \text { C19B-C18B-H18D } & 110.7 \\ \text { C17B-C18B-H18D } & 110.8 \\ \text { H18C-C18B-H18D } & 108.9 \\ \text { C20B-C19B-C18B } & 107.17(18) \\ \text { C20B-C19B-H19C } & 110.3 \\ \text { C18B-C19B-H19C } & 110.3 \\ \text { C20B-C19B-H19D } & 110.3 \\ \text { C18B-C19B-H19D } & 110.3 \\ \text { H19C-C19B-H19D } & 108.5 \\ \text { C18B-C19C-C20B } & 101.5(5) \\ \text { C18B-C19C-H19E } & 111.5 \\ \text { C20B-C19C-H19E } & 111.5 \\ \text { C18B-C19C-H19F } & 111.5 \\ \text { C20B-C19C-H19F } & 111.5 \\ \text { H19E-C19C-H19F } & 109.3 \\ \text { C19B-C20B-C21B } & 106.71(15) \\ \text { C19B-C20B-C19C } & 28.3(3) \\ \text { C21B-C20B-C19C } & 97.0(3) \\ \text { C19B-C20B-H20C } & 110.6 \\ & \end{array}$




\begin{tabular}{|c|c|}
\hline $\mathrm{C} 20 \mathrm{~A}-\mathrm{C} 21 \mathrm{~A}-\mathrm{C} 17 \mathrm{~A}$ & $103.98(12)$ \\
\hline $\mathrm{C} 20 \mathrm{~A}-\mathrm{C} 21 \mathrm{~A}-\mathrm{H} 21 \mathrm{~A}$ & 111.0 \\
\hline $\mathrm{C} 17 \mathrm{~A}-\mathrm{C} 21 \mathrm{~A}-\mathrm{H} 21 \mathrm{~A}$ & 111.0 \\
\hline $\mathrm{C} 20 \mathrm{~A}-\mathrm{C} 21 \mathrm{~A}-\mathrm{H} 21 \mathrm{~B}$ & 111.0 \\
\hline $\mathrm{C} 17 \mathrm{~A}-\mathrm{C} 21 \mathrm{~A}-\mathrm{H} 21 \mathrm{~B}$ & 111.0 \\
\hline $\mathrm{H} 21 \mathrm{~A}-\mathrm{C} 21 \mathrm{~A}-\mathrm{H} 21 \mathrm{~B}$ & 109.0 \\
\hline $\mathrm{O} 5 \mathrm{~A}-\mathrm{C} 22 \mathrm{~A}-\mathrm{N} 3 \mathrm{~A}$ & $123.32(14)$ \\
\hline $\mathrm{O} 5 \mathrm{~A}-\mathrm{C} 22 \mathrm{~A}-\mathrm{C} 17 \mathrm{~A}$ & $119.38(13)$ \\
\hline $\mathrm{N} 3 \mathrm{~A}-\mathrm{C} 22 \mathrm{~A}-\mathrm{C} 17 \mathrm{~A}$ & $117.21(13)$ \\
\hline $\mathrm{N} 3 \mathrm{~A}-\mathrm{C} 23 \mathrm{~A}-\mathrm{C} 28 \mathrm{~A}$ & $111.96(13)$ \\
\hline $\mathrm{N} 3 \mathrm{~A}-\mathrm{C} 23 \mathrm{~A}-\mathrm{C} 24 \mathrm{~A}$ & $110.06(13)$ \\
\hline $\mathrm{C} 28 \mathrm{~A}-\mathrm{C} 23 \mathrm{~A}-\mathrm{C} 24 \mathrm{~A}$ & $110.42(14)$ \\
\hline $\mathrm{N} 3 \mathrm{~A}-\mathrm{C} 23 \mathrm{~A}-\mathrm{H} 23 \mathrm{~A}$ & 108.1 \\
\hline $\mathrm{C} 28 \mathrm{~A}-\mathrm{C} 23 \mathrm{~A}-\mathrm{H} 23 \mathrm{~A}$ & 108.1 \\
\hline $\mathrm{C} 24 \mathrm{~A}-\mathrm{C} 23 \mathrm{~A}-\mathrm{H} 23 \mathrm{~A}$ & 108.1 \\
\hline $\mathrm{C} 25 \mathrm{~A}-\mathrm{C} 24 \mathrm{~A}-\mathrm{C} 23 \mathrm{~A}$ & $110.56(14)$ \\
\hline $\mathrm{C} 25 \mathrm{~A}-\mathrm{C} 24 \mathrm{~A}-\mathrm{H} 24 \mathrm{~B}$ & 109.5 \\
\hline $\mathrm{C} 23 \mathrm{~A}-\mathrm{C} 24 \mathrm{~A}-\mathrm{H} 24 \mathrm{~B}$ & 109.5 \\
\hline $\mathrm{C} 25 \mathrm{~A}-\mathrm{C} 24 \mathrm{~A}-\mathrm{H} 24 \mathrm{C}$ & 109.5 \\
\hline $\mathrm{C} 23 \mathrm{~A}-\mathrm{C} 24 \mathrm{~A}-\mathrm{H} 24 \mathrm{C}$ & 109.5 \\
\hline $\mathrm{H} 24 \mathrm{~B}-\mathrm{C} 24 \mathrm{~A}-\mathrm{H} 24 \mathrm{C}$ & 108.1 \\
\hline $\mathrm{C} 26 \mathrm{~A}-\mathrm{C} 25 \mathrm{~A}-\mathrm{C} 24 \mathrm{~A}$ & $110.86(15)$ \\
\hline $\mathrm{C} 26 \mathrm{~A}-\mathrm{C} 25 \mathrm{~A}-\mathrm{H} 25 \mathrm{~A}$ & 109.5 \\
\hline $\mathrm{C} 24 \mathrm{~A}-\mathrm{C} 25 \mathrm{~A}-\mathrm{H} 25 \mathrm{~A}$ & 109.5 \\
\hline $\mathrm{C} 26 \mathrm{~A}-\mathrm{C} 25 \mathrm{~A}-\mathrm{H} 25 \mathrm{~B}$ & 109.5 \\
\hline $\mathrm{C} 24 \mathrm{~A}-\mathrm{C} 25 \mathrm{~A}-\mathrm{H} 25 \mathrm{~B}$ & 109.5 \\
\hline $\mathrm{H} 25 \mathrm{~A}-\mathrm{C} 25 \mathrm{~A}-\mathrm{H} 25 \mathrm{~B}$ & 108.1 \\
\hline $\mathrm{C} 27 \mathrm{~A}-\mathrm{C} 26 \mathrm{~A}-\mathrm{C} 25 \mathrm{~A}$ & $111.34(15)$ \\
\hline $\mathrm{C} 27 \mathrm{~A}-\mathrm{C} 26 \mathrm{~A}-\mathrm{H} 26 \mathrm{~A}$ & 109.4 \\
\hline $\mathrm{C} 25 \mathrm{~A}-\mathrm{C} 26 \mathrm{~A}-\mathrm{H} 26 \mathrm{~A}$ & 109.4 \\
\hline $\mathrm{C} 27 \mathrm{~A}-\mathrm{C} 26 \mathrm{~A}-\mathrm{H} 26 \mathrm{~B}$ & 109.4 \\
\hline $\mathrm{C} 25 \mathrm{~A}-\mathrm{C} 26 \mathrm{~A}-\mathrm{H} 26 \mathrm{~B}$ & 109.4 \\
\hline $\mathrm{H} 26 \mathrm{~A}-\mathrm{C} 26 \mathrm{~A}-\mathrm{H} 26 \mathrm{~B}$ & 108.0 \\
\hline $\mathrm{C} 26 \mathrm{~A}-\mathrm{C} 27 \mathrm{~A}-\mathrm{C} 28 \mathrm{~A}$ & $111.77(15)$ \\
\hline $\mathrm{C} 26 \mathrm{~A}-\mathrm{C} 27 \mathrm{~A}-\mathrm{H} 27 \mathrm{~A}$ & 109.3 \\
\hline $\mathrm{C} 28 \mathrm{~A}-\mathrm{C} 27 \mathrm{~A}-\mathrm{H} 27 \mathrm{~A}$ & 109.3 \\
\hline $\mathrm{C} 26 \mathrm{~A}-\mathrm{C} 27 \mathrm{~A}-\mathrm{H} 27 \mathrm{~B}$ & 109.3 \\
\hline $\mathrm{C} 28 \mathrm{~A}-\mathrm{C} 27 \mathrm{~A}-\mathrm{H} 27 \mathrm{~B}$ & 109.3 \\
\hline $\mathrm{H} 27 \mathrm{~A}-\mathrm{C} 27 \mathrm{~A}-\mathrm{H} 27 \mathrm{~B}$ & 107.9 \\
\hline $\mathrm{C} 23 \mathrm{~A}-\mathrm{C} 28 \mathrm{~A}-\mathrm{C} 27 \mathrm{~A}$ & $110.50(15)$ \\
\hline $\mathrm{C} 23 \mathrm{~A}-\mathrm{C} 28 \mathrm{~A}-\mathrm{H} 28 \mathrm{~A}$ & 109.5 \\
\hline $\mathrm{C} 27 \mathrm{~A}-\mathrm{C} 28 \mathrm{~A}-\mathrm{H} 28 \mathrm{~A}$ & 109.5 \\
\hline $\mathrm{C} 23 \mathrm{~A}-\mathrm{C} 28 \mathrm{~A}-\mathrm{H} 28 \mathrm{~B}$ & 109.5 \\
\hline $\mathrm{C} 27 \mathrm{~A}-\mathrm{C} 28 \mathrm{~A}-\mathrm{H} 28 \mathrm{~B}$ & 109.5 \\
\hline $\mathrm{H} 28 \mathrm{~A}-\mathrm{C} 28 \mathrm{~A}-\mathrm{H} 28 \mathrm{~B}$ & 108.1 \\
\hline $\mathrm{C} 2 \mathrm{~B}-\mathrm{O} 1 \mathrm{~B}-\mathrm{N} 1 \mathrm{~B}$ & $108.16(11)$ \\
\hline $\mathrm{C} 3 \mathrm{~B}-\mathrm{N} 1 \mathrm{~B}-\mathrm{O} 1 \mathrm{~B}$ & $111.39(12)$ \\
\hline $\mathrm{C} 3 \mathrm{~B}-\mathrm{N} 1 \mathrm{~B}-\mathrm{C} 4 \mathrm{~B}$ & $135.91(13)$ \\
\hline
\end{tabular}

\begin{tabular}{|c|c|}
\hline $\mathrm{C} 21 \mathrm{~B}-\mathrm{C} 20 \mathrm{~B}-\mathrm{H} 20 \mathrm{C}$ & 110.2 \\
\hline $\mathrm{C} 19 \mathrm{C}-\mathrm{C} 20 \mathrm{~B}-\mathrm{H} 20 \mathrm{C}$ & 137.9 \\
\hline $\mathrm{C} 19 \mathrm{~B}-\mathrm{C} 20 \mathrm{~B}-\mathrm{H} 20 \mathrm{D}$ & 110.3 \\
\hline $\mathrm{C} 21 \mathrm{~B}-\mathrm{C} 20 \mathrm{~B}-\mathrm{H} 20 \mathrm{D}$ & 110.4 \\
\hline $\mathrm{C} 19 \mathrm{C}-\mathrm{C} 20 \mathrm{~B}-\mathrm{H} 20 \mathrm{D}$ & 90.0 \\
\hline $\mathrm{H} 20 \mathrm{C}-\mathrm{C} 20 \mathrm{~B}-\mathrm{H} 20 \mathrm{D}$ & 108.6 \\
\hline $\mathrm{C} 20 \mathrm{~B}-\mathrm{C} 21 \mathrm{~B}-\mathrm{C} 17 \mathrm{~B}$ & $105.29(13)$ \\
\hline $\mathrm{C} 20 \mathrm{~B}-\mathrm{C} 21 \mathrm{~B}-\mathrm{H} 21 \mathrm{C}$ & 110.7 \\
\hline $\mathrm{C} 17 \mathrm{~B}-\mathrm{C} 21 \mathrm{~B}-\mathrm{H} 21 \mathrm{C}$ & 110.7 \\
\hline $\mathrm{C} 20 \mathrm{~B}-\mathrm{C} 21 \mathrm{~B}-\mathrm{H} 21 \mathrm{D}$ & 110.7 \\
\hline $\mathrm{C} 17 \mathrm{~B}-\mathrm{C} 21 \mathrm{~B}-\mathrm{H} 21 \mathrm{D}$ & 110.7 \\
\hline $\mathrm{H} 21 \mathrm{C}-\mathrm{C} 21 \mathrm{~B}-\mathrm{H} 21 \mathrm{D}$ & 108.8 \\
\hline $\mathrm{O} 5 \mathrm{~B}-\mathrm{C} 22 \mathrm{~B}-\mathrm{N} 3 \mathrm{~B}$ & $122.59(14)$ \\
\hline $\mathrm{O} 5 \mathrm{~B}-\mathrm{C} 22 \mathrm{~B}-\mathrm{C} 17 \mathrm{~B}$ & $119.66(14)$ \\
\hline $\mathrm{N} 3 \mathrm{~B}-\mathrm{C} 22 \mathrm{~B}-\mathrm{C} 17 \mathrm{~B}$ & $117.71(13)$ \\
\hline $\mathrm{N} 3 \mathrm{~B}-\mathrm{C} 23 \mathrm{~B}-\mathrm{C} 24 \mathrm{~B}$ & $110.78(13)$ \\
\hline $\mathrm{N} 3 \mathrm{~B}-\mathrm{C} 23 \mathrm{~B}-\mathrm{C} 28 \mathrm{~B}$ & $111.91(13)$ \\
\hline $\mathrm{C} 24 \mathrm{~B}-\mathrm{C} 23 \mathrm{~B}-\mathrm{C} 28 \mathrm{~B}$ & $110.06(13)$ \\
\hline $\mathrm{N} 3 \mathrm{~B}-\mathrm{C} 23 \mathrm{~B}-\mathrm{H} 23 \mathrm{~B}$ & 108.0 \\
\hline $\mathrm{C} 24 \mathrm{~B}-\mathrm{C} 23 \mathrm{~B}-\mathrm{H} 23 \mathrm{~B}$ & 108.0 \\
\hline $\mathrm{C} 28 \mathrm{~B}-\mathrm{C} 23 \mathrm{~B}-\mathrm{H} 23 \mathrm{~B}$ & 108.0 \\
\hline $\mathrm{C} 23 \mathrm{~B}-\mathrm{C} 24 \mathrm{~B}-\mathrm{C} 25 \mathrm{~B}$ & $109.54(13)$ \\
\hline $\mathrm{C} 23 \mathrm{~B}-\mathrm{C} 24 \mathrm{~B}-\mathrm{H} 24 \mathrm{~A}$ & 109.8 \\
\hline $\mathrm{C} 25 \mathrm{~B}-\mathrm{C} 24 \mathrm{~B}-\mathrm{H} 24 \mathrm{~A}$ & 109.8 \\
\hline $\mathrm{C} 23 \mathrm{~B}-\mathrm{C} 24 \mathrm{~B}-\mathrm{H} 24 \mathrm{D}$ & 109.8 \\
\hline $\mathrm{C} 25 \mathrm{~B}-\mathrm{C} 24 \mathrm{~B}-\mathrm{H} 24 \mathrm{D}$ & 109.8 \\
\hline $\mathrm{H} 24 \mathrm{~A}-\mathrm{C} 24 \mathrm{~B}-\mathrm{H} 24 \mathrm{D}$ & 108.2 \\
\hline $\mathrm{C} 26 \mathrm{~B}-\mathrm{C} 25 \mathrm{~B}-\mathrm{C} 24 \mathrm{~B}$ & $110.44(13)$ \\
\hline $\mathrm{C} 26 \mathrm{~B}-\mathrm{C} 25 \mathrm{~B}-\mathrm{H} 25 \mathrm{C}$ & 109.6 \\
\hline $\mathrm{C} 24 \mathrm{~B}-\mathrm{C} 25 \mathrm{~B}-\mathrm{H} 25 \mathrm{C}$ & 109.6 \\
\hline $\mathrm{C} 26 \mathrm{~B}-\mathrm{C} 25 \mathrm{~B}-\mathrm{H} 25 \mathrm{D}$ & 109.6 \\
\hline $\mathrm{C} 24 \mathrm{~B}-\mathrm{C} 25 \mathrm{~B}-\mathrm{H} 25 \mathrm{D}$ & 109.6 \\
\hline $\mathrm{H} 25 \mathrm{C}-\mathrm{C} 25 \mathrm{~B}-\mathrm{H} 25 \mathrm{D}$ & 108.1 \\
\hline $\mathrm{C} 25 \mathrm{~B}-\mathrm{C} 26 \mathrm{~B}-\mathrm{C} 27 \mathrm{~B}$ & $111.46(14)$ \\
\hline $\mathrm{C} 25 \mathrm{~B}-\mathrm{C} 26 \mathrm{~B}-\mathrm{H} 26 \mathrm{C}$ & 109.3 \\
\hline $\mathrm{C} 27 \mathrm{~B}-\mathrm{C} 26 \mathrm{~B}-\mathrm{H} 26 \mathrm{C}$ & 109.3 \\
\hline $\mathrm{C} 25 \mathrm{~B}-\mathrm{C} 26 \mathrm{~B}-\mathrm{H} 26 \mathrm{D}$ & 109.3 \\
\hline $\mathrm{C} 27 \mathrm{~B}-\mathrm{C} 26 \mathrm{~B}-\mathrm{H} 26 \mathrm{D}$ & 109.3 \\
\hline $\mathrm{H} 26 \mathrm{C}-\mathrm{C} 26 \mathrm{~B}-\mathrm{H} 26 \mathrm{D}$ & 108.0 \\
\hline $\mathrm{C} 26 \mathrm{~B}-\mathrm{C} 27 \mathrm{~B}-\mathrm{C} 28 \mathrm{~B}$ & $112.36(15)$ \\
\hline $\mathrm{C} 26 \mathrm{~B}-\mathrm{C} 27 \mathrm{~B}-\mathrm{H} 27 \mathrm{C}$ & 109.1 \\
\hline $\mathrm{C} 28 \mathrm{~B}-\mathrm{C} 27 \mathrm{~B}-\mathrm{H} 27 \mathrm{C}$ & 109.1 \\
\hline $\mathrm{C} 26 \mathrm{~B}-\mathrm{C} 27 \mathrm{~B}-\mathrm{H} 27 \mathrm{D}$ & 109.1 \\
\hline $\mathrm{C} 28 \mathrm{~B}-\mathrm{C} 27 \mathrm{~B}-\mathrm{H} 27 \mathrm{D}$ & 109.1 \\
\hline $\mathrm{H} 27 \mathrm{C}-\mathrm{C} 27 \mathrm{~B}-\mathrm{H} 27 \mathrm{D}$ & 107.9 \\
\hline $\mathrm{C} 23 \mathrm{~B}-\mathrm{C} 28 \mathrm{~B}-\mathrm{C} 27 \mathrm{~B}$ & $110.37(14)$ \\
\hline $\mathrm{C} 23 \mathrm{~B}-\mathrm{C} 28 \mathrm{~B}-\mathrm{H} 28 \mathrm{C}$ & 109.6 \\
\hline $\mathrm{C} 27 \mathrm{~B}-\mathrm{C} 28 \mathrm{~B}-\mathrm{H} 28 \mathrm{C}$ & 109.6 \\
\hline
\end{tabular}




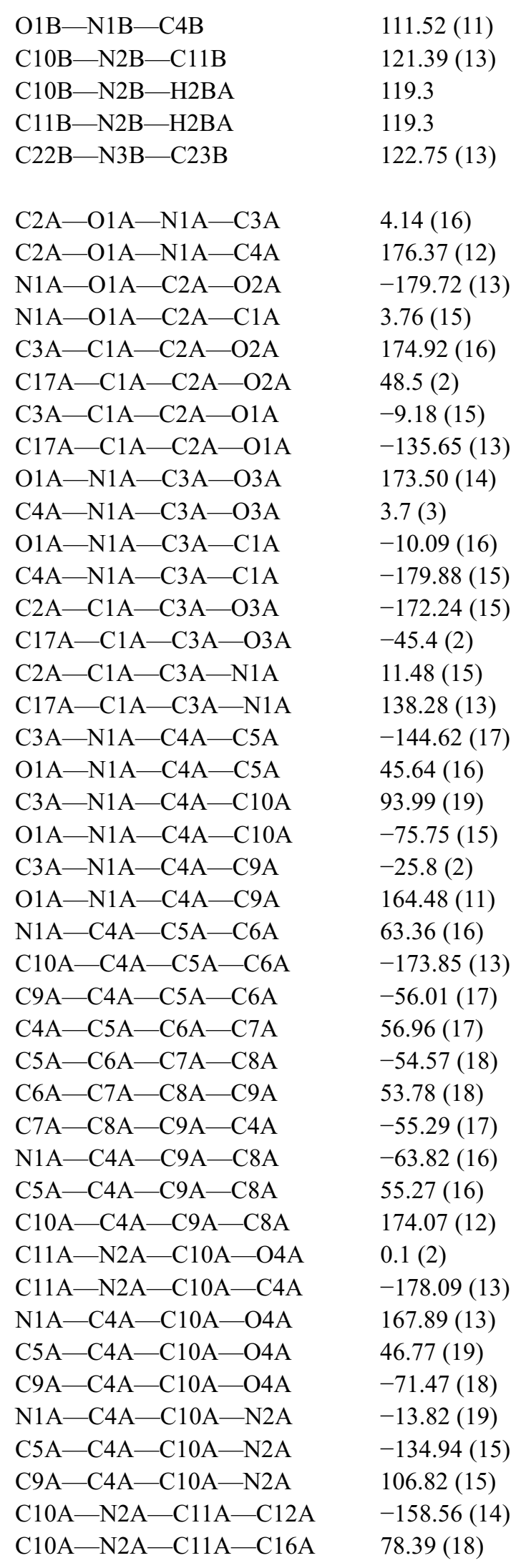

$111.52(11)$
$121.39(13)$
119.3
119.3
$122.75(13)$

$4.14(16)$

176.37 (12)

$-179.72(13)$

$3.76(15)$

$174.92(16)$

48.5 (2)

$-9.18(15)$

$-135.65(13)$

$173.50(14)$

3.7 (3)

$-10.09(16)$

$-179.88(15)$

$-172.24(15)$

$-45.4(2)$

$11.48(15)$

$138.28(13)$

$-144.62(17)$

$45.64(16)$

93.99 (19)

$-75.75(15)$

-25.8 (2)

164.48 (11)

$63.36(16)$

$-173.85(13)$

$-56.01(17)$

56.96 (17)

$-54.57(18)$

$53.78(18)$

$-55.29(17)$

$-63.82(16)$

$55.27(16)$

174.07 (12)

0.1 (2)

-178.09 (13)

$167.89(13)$

46.77 (19)

$-71.47(18)$

$-13.82(19)$

-134.94 (15)

$106.82(15)$

$-158.56(14)$

78.39 (18)

C23B-C28B-H28D
C27B-C28B-H28D
H28C-C28B-H28D
H1WA-O1W-H1WB

109.6

109.6

108.1

$106(2)$

$\begin{array}{ll}\mathrm{C} 17 \mathrm{~B}-\mathrm{C} 1 \mathrm{~B}-\mathrm{C} 2 \mathrm{~B}-\mathrm{O} 2 \mathrm{~B} & 47.6(2) \\ \mathrm{C} 3 \mathrm{~B}-\mathrm{C} 1 \mathrm{~B}-\mathrm{C} 2 \mathrm{~B}-\mathrm{O} 1 \mathrm{~B} & -10.92(16) \\ \mathrm{C} 17 \mathrm{~B}-\mathrm{C} 1 \mathrm{~B}-\mathrm{C} 2 \mathrm{~B}-\mathrm{O} 1 \mathrm{~B} & -136.68(13) \\ \mathrm{O} 1 \mathrm{~B}-\mathrm{N} 1 \mathrm{~B}-\mathrm{C} 3 \mathrm{~B}-\mathrm{O} 3 \mathrm{~B} & 171.91(14) \\ \mathrm{C} 4 \mathrm{~B}-\mathrm{N} 1 \mathrm{~B}-\mathrm{C} 3 \mathrm{~B}-\mathrm{O} 3 \mathrm{~B} & 5.9(3) \\ \mathrm{O} 1 \mathrm{~B}-\mathrm{N} 1 \mathrm{~B}-\mathrm{C} 3 \mathrm{~B}-\mathrm{C} 1 \mathrm{~B} & -10.74(16) \\ \mathrm{C} 4 \mathrm{~B}-\mathrm{N} 1 \mathrm{~B}-\mathrm{C} 3 \mathrm{~B}-\mathrm{C} 1 \mathrm{~B} & -176.79(15) \\ \mathrm{C} 2 \mathrm{~B}-\mathrm{C} 1 \mathrm{~B}-\mathrm{C} 3 \mathrm{~B}-\mathrm{O} 3 \mathrm{~B} & -169.86(15)\end{array}$

$\mathrm{C} 17 \mathrm{~B}-\mathrm{C} 1 \mathrm{~B}-\mathrm{C} 3 \mathrm{~B}-\mathrm{O} 3 \mathrm{~B} \quad-43.6$ (2)

$\mathrm{C} 2 \mathrm{~B}-\mathrm{C} 1 \mathrm{~B}-\mathrm{C} 3 \mathrm{~B}-\mathrm{N} 1 \mathrm{~B}$

$\mathrm{C} 17 \mathrm{~B}-\mathrm{C} 1 \mathrm{~B}-\mathrm{C} 3 \mathrm{~B}-\mathrm{N} 1 \mathrm{~B}$

$\mathrm{C} 3 \mathrm{~B}-\mathrm{N} 1 \mathrm{~B}-\mathrm{C} 4 \mathrm{~B}-\mathrm{C} 5 \mathrm{~B}$

$\mathrm{O} 1 \mathrm{~B}-\mathrm{N} 1 \mathrm{~B}-\mathrm{C} 4 \mathrm{~B}-\mathrm{C} 5 \mathrm{~B}$

$\mathrm{C} 3 \mathrm{~B}-\mathrm{N} 1 \mathrm{~B}-\mathrm{C} 4 \mathrm{~B}-\mathrm{C} 10 \mathrm{~B}$

$\mathrm{O} 1 \mathrm{~B}-\mathrm{N} 1 \mathrm{~B}-\mathrm{C} 4 \mathrm{~B}-\mathrm{C} 10 \mathrm{~B}$

$\mathrm{C} 3 \mathrm{~B}-\mathrm{N} 1 \mathrm{~B}-\mathrm{C} 4 \mathrm{~B}-\mathrm{C} 9 \mathrm{~B}$

$\mathrm{O} 1 \mathrm{~B}-\mathrm{N} 1 \mathrm{~B}-\mathrm{C} 4 \mathrm{~B}-\mathrm{C} 9 \mathrm{~B}$

$\mathrm{N} 1 \mathrm{~B}-\mathrm{C} 4 \mathrm{~B}-\mathrm{C} 5 \mathrm{~B}-\mathrm{C} 6 \mathrm{~B}$

$\mathrm{C} 10 \mathrm{~B}-\mathrm{C} 4 \mathrm{~B}-\mathrm{C} 5 \mathrm{~B}-\mathrm{C} 6 \mathrm{~B}$

$\mathrm{C} 9 \mathrm{~B}-\mathrm{C} 4 \mathrm{~B}-\mathrm{C} 5 \mathrm{~B}-\mathrm{C} 6 \mathrm{~B}$

$\mathrm{C} 4 \mathrm{~B}-\mathrm{C} 5 \mathrm{~B}-\mathrm{C} 6 \mathrm{~B}-\mathrm{C} 7 \mathrm{~B}$

$\mathrm{C} 5 \mathrm{~B}-\mathrm{C} 6 \mathrm{~B}-\mathrm{C} 7 \mathrm{~B}-\mathrm{C} 8 \mathrm{~B}$

$\mathrm{C} 6 \mathrm{~B}-\mathrm{C} 7 \mathrm{~B}-\mathrm{C} 8 \mathrm{~B}-\mathrm{C} 9 \mathrm{~B}$

$\mathrm{C} 7 \mathrm{~B}-\mathrm{C} 8 \mathrm{~B}-\mathrm{C} 9 \mathrm{~B}-\mathrm{C} 4 \mathrm{~B}$

$\mathrm{N} 1 \mathrm{~B}-\mathrm{C} 4 \mathrm{~B}-\mathrm{C} 9 \mathrm{~B}-\mathrm{C} 8 \mathrm{~B}$

$\mathrm{C} 5 \mathrm{~B}-\mathrm{C} 4 \mathrm{~B}-\mathrm{C} 9 \mathrm{~B}-\mathrm{C} 8 \mathrm{~B}$

$\mathrm{C} 10 \mathrm{~B}-\mathrm{C} 4 \mathrm{~B}-\mathrm{C} 9 \mathrm{~B}-\mathrm{C} 8 \mathrm{~B}$

$\mathrm{C} 11 \mathrm{~B}-\mathrm{N} 2 \mathrm{~B}-\mathrm{C} 10 \mathrm{~B}-\mathrm{O} 4 \mathrm{~B}$

$\mathrm{C} 11 \mathrm{~B}-\mathrm{N} 2 \mathrm{~B}-\mathrm{C} 10 \mathrm{~B}-\mathrm{C} 4 \mathrm{~B}$

$\mathrm{N} 1 \mathrm{~B}-\mathrm{C} 4 \mathrm{~B}-\mathrm{C} 10 \mathrm{~B}-\mathrm{O} 4 \mathrm{~B}$

$\mathrm{C} 5 \mathrm{~B}-\mathrm{C} 4 \mathrm{~B}-\mathrm{C} 10 \mathrm{~B}-\mathrm{O} 4 \mathrm{~B}$

$\mathrm{C} 9 \mathrm{~B}-\mathrm{C} 4 \mathrm{~B}-\mathrm{C} 10 \mathrm{~B}-\mathrm{O} 4 \mathrm{~B}$

$\mathrm{N} 1 \mathrm{~B}-\mathrm{C} 4 \mathrm{~B}-\mathrm{C} 10 \mathrm{~B}-\mathrm{N} 2 \mathrm{~B}$

$\mathrm{C} 5 \mathrm{~B}-\mathrm{C} 4 \mathrm{~B}-\mathrm{C} 10 \mathrm{~B}-\mathrm{N} 2 \mathrm{~B}$

$\mathrm{C} 9 \mathrm{~B}-\mathrm{C} 4 \mathrm{~B}-\mathrm{C} 10 \mathrm{~B}-\mathrm{N} 2 \mathrm{~B}$

$\mathrm{C} 10 \mathrm{~B}-\mathrm{N} 2 \mathrm{~B}-\mathrm{C} 11 \mathrm{~B}-\mathrm{C} 12 \mathrm{~B}$

$\mathrm{C} 10 \mathrm{~B}-\mathrm{N} 2 \mathrm{~B}-\mathrm{C} 11 \mathrm{~B}-\mathrm{C} 16 \mathrm{~B}$

$\mathrm{N} 2 \mathrm{~B}-\mathrm{C} 11 \mathrm{~B}-\mathrm{C} 12 \mathrm{~B}-\mathrm{C} 13 \mathrm{~B}$

$\mathrm{C} 16 \mathrm{~B}-\mathrm{C} 11 \mathrm{~B}-\mathrm{C} 12 \mathrm{~B}-\mathrm{C} 13 \mathrm{~B}$

$\mathrm{C} 11 \mathrm{~B}-\mathrm{C} 12 \mathrm{~B}-\mathrm{C} 13 \mathrm{~B}-\mathrm{C} 14 \mathrm{~B}$

C12B $-\mathrm{C} 13 \mathrm{~B}-\mathrm{C} 14 \mathrm{~B}-\mathrm{C} 15 \mathrm{~B}$

$\mathrm{C} 13 \mathrm{~B}-\mathrm{C} 14 \mathrm{~B}-\mathrm{C} 15 \mathrm{~B}-\mathrm{C} 16 \mathrm{~B}$
$12.87(15)$

$139.12(13)$

$-143.84(17)$

$50.13(16)$

$93.8(2)$

$-72.23(15)$

$-24.3(2)$

$169.71(11)$

66.97 (17)

$-169.16(14)$

$-52.95(18)$

56.25 (19)

-56.50 (19)

55.40 (19)

-54.89 (19)

$-66.83(17)$

52.77 (18)

$171.30(13)$

3.4 (2)

$-172.06(13)$

$161.28(14)$

$39.4(2)$

$-78.65(18)$

-23.0 (2)

$-144.92(15)$

97.08 (16)

$-160.56(14)$

$76.50(19)$

$179.36(13)$

$-57.53(18)$

$58.15(19)$

$-57.6(2)$

$56.3(2)$ 


\begin{tabular}{|c|c|}
\hline 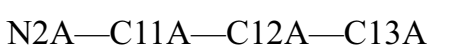 & 179.6 \\
\hline $\mathrm{C} 16 \mathrm{~A}-\mathrm{C} 11 \mathrm{~A}-\mathrm{C} 12 \mathrm{~A}-\mathrm{C} 13 \mathrm{~A}$ & $-56.80(18)$ \\
\hline $\mathrm{C} 11 \mathrm{~A}-\mathrm{C} 12 \mathrm{~A}-\mathrm{C} 13 \mathrm{~A}-\mathrm{C} 14 \mathrm{~A}$ & $56.2(2)$ \\
\hline $\mathrm{C} 12 \mathrm{~A}-\mathrm{C} 13 \mathrm{~A}-\mathrm{C} 14 \mathrm{~A}-\mathrm{C} 15 \mathrm{~A}$ & $-55.4(2)$ \\
\hline $\mathrm{C} 13 \mathrm{~A}-\mathrm{C} 14 \mathrm{~A}-\mathrm{C} 15 \mathrm{~A}-\mathrm{C} 16 \mathrm{~A}$ & $55.9(2)$ \\
\hline $\mathrm{N} 2 \mathrm{~A}-\mathrm{C} 11 \mathrm{~A}-\mathrm{C} 16 \mathrm{~A}-\mathrm{C} 15 \mathrm{~A}$ & $179.15(14)$ \\
\hline $\mathrm{C} 12 \mathrm{~A}-\mathrm{C} 11 \mathrm{~A}-\mathrm{C} 16 \mathrm{~A}-\mathrm{C} 15 \mathrm{~A}$ & $56.66(19)$ \\
\hline $\mathrm{C} 14 \mathrm{~A}-\mathrm{C} 15 \mathrm{~A}-\mathrm{C} 16 \mathrm{~A}-\mathrm{C} 11 \mathrm{~A}$ & $-56.4(2)$ \\
\hline $\mathrm{C} 2 \mathrm{~A}-\mathrm{C} 1 \mathrm{~A}-\mathrm{C} 17 \mathrm{~A}-\mathrm{C} 22 \mathrm{~A}$ & $61.15(16)$ \\
\hline $\mathrm{C} 3 \mathrm{~A}-\mathrm{C} 1 \mathrm{~A}-\mathrm{C} 17 \mathrm{~A}-\mathrm{C} 22 \mathrm{~A}$ & (16) \\
\hline $\mathrm{C} 2 \mathrm{~A}-\mathrm{C} 1 \mathrm{~A}-\mathrm{C} 17 \mathrm{~A}-\mathrm{C} 18 \mathrm{~A}$ & $-172.21(12)$ \\
\hline $\mathrm{C} 3 \mathrm{~A}-\mathrm{C} 1 \mathrm{~A}-\mathrm{C} 17 \mathrm{~A}-\mathrm{C} 18 \mathrm{~A}$ & \\
\hline $\mathrm{C} 2 \mathrm{~A}-\mathrm{C} 1 \mathrm{~A}-\mathrm{C} 17 \mathrm{~A}-\mathrm{C} 21 \mathrm{~A}$ & -6 \\
\hline $\mathrm{C} 3 \mathrm{~A}-\mathrm{C} 1 \mathrm{~A}-\mathrm{C} 17 \mathrm{~A}-\mathrm{C} 21 \mathrm{~A}$ & \\
\hline $\mathrm{C} 22 \mathrm{~A}-\mathrm{C} 17 \mathrm{~A}-\mathrm{C} 18 \mathrm{~A}-\mathrm{C} 19 \mathrm{~A}$ & \\
\hline $\mathrm{C} 1 \mathrm{~A}-\mathrm{C} 17 \mathrm{~A}-\mathrm{C} 18 \mathrm{~A}-\mathrm{C} 19 \mathrm{~A}$ & \\
\hline $\mathrm{C} 21 \mathrm{~A}-\mathrm{C} 17 \mathrm{~A}-\mathrm{C} 18 \mathrm{~A}-\mathrm{C} 19 \mathrm{~A}$ & $-3 c$ \\
\hline $\mathrm{C} 17 \mathrm{~A}-\mathrm{C} 18 \mathrm{~A}-\mathrm{C} 19 \mathrm{~A}-\mathrm{C} 20 \mathrm{~A}$ & \\
\hline $\mathrm{C} 18 \mathrm{~A}-\mathrm{C} 19 \mathrm{~A}-\mathrm{C} 20 \mathrm{~A}-\mathrm{C} 21 \mathrm{~A}$ & \\
\hline $\mathrm{C} 19 \mathrm{~A}-\mathrm{C} 20 \mathrm{~A}-\mathrm{C} 21 \mathrm{~A}-\mathrm{C} 17 \mathrm{~A}$ & 17) \\
\hline $\mathrm{C} 22 \mathrm{~A}-\mathrm{C} 17 \mathrm{~A}-\mathrm{C} 21 \mathrm{~A}-\mathrm{C} 20 \mathrm{~A}$ & 16 \\
\hline $\mathrm{C} 1 \mathrm{~A}-\mathrm{C} 17 \mathrm{~A}-\mathrm{C} 21 \mathrm{~A}-\mathrm{C} 20 \mathrm{~A}$ & 15) \\
\hline $\mathrm{C} 18 \mathrm{~A}-\mathrm{C} 17 \mathrm{~A}-\mathrm{C} 21 \mathrm{~A}-\mathrm{C} 20 \mathrm{~A}$ & \\
\hline $\mathrm{C} 23 \mathrm{~A}-\mathrm{N} 3 \mathrm{~A}-\mathrm{C} 22 \mathrm{~A}-\mathrm{O} 5 \mathrm{~A}$ & -5 \\
\hline $\mathrm{C} 23 \mathrm{~A}-\mathrm{N} 3 \mathrm{~A}-\mathrm{C} 22 \mathrm{~A}-\mathrm{C} 17 \mathrm{~A}$ & 17 \\
\hline $\mathrm{C} 1 \mathrm{~A}-\mathrm{C} 17 \mathrm{~A}-\mathrm{C} 22 \mathrm{~A}-\mathrm{O} 5 \mathrm{~A}$ & \\
\hline $\mathrm{C} 18 \mathrm{~A}-\mathrm{C} 17 \mathrm{~A}-\mathrm{C} 22 \mathrm{~A}-\mathrm{O} 5 \mathrm{~A}$ & \\
\hline $\mathrm{C} 21 \mathrm{~A}-\mathrm{C} 17 \mathrm{~A}-\mathrm{C} 22 \mathrm{~A}-\mathrm{O} 5 \mathrm{~A}$ & 11 \\
\hline$-\mathrm{C} 22 \mathrm{~A}-\mathrm{N} 3 \mathrm{~A}$ & 3) \\
\hline $\mathrm{C} 18 \mathrm{~A}-\mathrm{C} 17 \mathrm{~A}-\mathrm{C} 22 \mathrm{~A}-\mathrm{N} 3 \mathrm{~A}$ & \\
\hline $\mathrm{C} 21 \mathrm{~A}-\mathrm{C} 17 \mathrm{~A}-\mathrm{C} 22 \mathrm{~A}-\mathrm{N} 3 \mathrm{~A}$ & -61 \\
\hline $\mathrm{C} 22 \mathrm{~A}-\mathrm{N} 3 \mathrm{~A}-\mathrm{C} 23 \mathrm{~A}-\mathrm{C} 28 \mathrm{~A}$ & 100 \\
\hline $\mathrm{C} 22 \mathrm{~A}-\mathrm{N} 3 \mathrm{~A}-\mathrm{C} 23 \mathrm{~A}-\mathrm{C} 24 \mathrm{~A}$ & -1 \\
\hline $\mathrm{N} 3 \mathrm{~A}-\mathrm{C} 23 \mathrm{~A}-\mathrm{C} 24 \mathrm{~A}-\mathrm{C} 25 \mathrm{~A}$ & 177 \\
\hline $\mathrm{C} 28 \mathrm{~A}-\mathrm{C} 23 \mathrm{~A}-\mathrm{C} 24 \mathrm{~A}-\mathrm{C} 25 \mathrm{~A}$ & $3(18)$ \\
\hline $\mathrm{C} 23 \mathrm{~A}-\mathrm{C} 24 \mathrm{~A}-\mathrm{C} 25 \mathrm{~A}-\mathrm{C} 26 \mathrm{~A}$ & 57.0 \\
\hline $\mathrm{C} 24 \mathrm{~A}-\mathrm{C} 25 \mathrm{~A}-\mathrm{C} 26 \mathrm{~A}-\mathrm{C} 27 \mathrm{~A}$ & -55 \\
\hline $\mathrm{C} 25 \mathrm{~A}-\mathrm{C} 26 \mathrm{~A}-\mathrm{C} 27 \mathrm{~A}-\mathrm{C} 28 \mathrm{~A}$ & $54.6(2)$ \\
\hline $\mathrm{N} 3 \mathrm{~A}-\mathrm{C} 23 \mathrm{~A}-\mathrm{C} 28 \mathrm{~A}-\mathrm{C} 27 \mathrm{~A}$ & -17 \\
\hline $\mathrm{C} 24 \mathrm{~A}-\mathrm{C} 23 \mathrm{~A}-\mathrm{C} 28 \mathrm{~A}-\mathrm{C} 27 \mathrm{~A}$ & $57.15(19)$ \\
\hline $\mathrm{C} 26 \mathrm{~A}-\mathrm{C} 27 \mathrm{~A}-\mathrm{C} 28 \mathrm{~A}-\mathrm{C} 23 \mathrm{~A}$ & $-55.5(2)$ \\
\hline $\mathrm{C} 2 \mathrm{~B}-\mathrm{O} 1 \mathrm{~B}-\mathrm{N} 1 \mathrm{~B}-\mathrm{C} 3 \mathrm{~B}$ & $3.71(16)$ \\
\hline $\mathrm{C} 2 \mathrm{~B}-\mathrm{O} 1 \mathrm{~B}-\mathrm{N} 1 \mathrm{~B}-\mathrm{C} 4 \mathrm{~B}$ & \\
\hline $\mathrm{N} 1 \mathrm{~B}-\mathrm{O} 1 \mathrm{~B}-\mathrm{C} 2 \mathrm{~B}-\mathrm{O} 2 \mathrm{~B}$ & $-178.50(1$ \\
\hline $\mathrm{N} 1 \mathrm{~B}-\mathrm{O} 1 \mathrm{~B}-\mathrm{C} 2 \mathrm{~B}-\mathrm{C} 1 \mathrm{~B}$ & $5.15(16)$ \\
\hline $\mathrm{C} 3 \mathrm{~B}-\mathrm{C} 1 \mathrm{~B}-\mathrm{C} 2 \mathrm{~B}-\mathrm{O} 2 \mathrm{~B}$ & $173.34(17)$ \\
\hline
\end{tabular}

\begin{tabular}{|c|c|}
\hline $\mathrm{C} 14 \mathrm{~B}-\mathrm{C} 15 \mathrm{~B}-\mathrm{C} 16 \mathrm{~B}-\mathrm{C} 11 \mathrm{~B}$ & $-55.4(2)$ \\
\hline $\mathrm{N} 2 \mathrm{~B}-\mathrm{C} 11 \mathrm{~B}-\mathrm{C} 16 \mathrm{~B}-\mathrm{C} 15 \mathrm{~B}$ & $178.77(14)$ \\
\hline $\mathrm{C} 12 \mathrm{~B}-\mathrm{C} 11 \mathrm{~B}-\mathrm{C} 16 \mathrm{~B}-\mathrm{C} 15 \mathrm{~B}$ & $56.24(19)$ \\
\hline $\mathrm{C} 2 \mathrm{~B}-\mathrm{C} 1 \mathrm{~B}-\mathrm{C} 17 \mathrm{~B}-\mathrm{C} 22 \mathrm{~B}$ & $59.88(17)$ \\
\hline $\mathrm{C} 3 \mathrm{~B}-\mathrm{C} 1 \mathrm{~B}-\mathrm{C} 17 \mathrm{~B}-\mathrm{C} 22 \mathrm{~B}$ & $-59.04(17)$ \\
\hline $\mathrm{C} 2 \mathrm{~B}-\mathrm{C} 1 \mathrm{~B}-\mathrm{C} 17 \mathrm{~B}-\mathrm{C} 18 \mathrm{~B}$ & $-174.71(13)$ \\
\hline $\mathrm{C} 3 \mathrm{~B}-\mathrm{C} 1 \mathrm{~B}-\mathrm{C} 17 \mathrm{~B}-\mathrm{C} 18 \mathrm{~B}$ & $66.37(17)$ \\
\hline $\mathrm{C} 2 \mathrm{~B}-\mathrm{C} 1 \mathrm{~B}-\mathrm{C} 17 \mathrm{~B}-\mathrm{C} 21 \mathrm{~B}$ & $-62.39(17)$ \\
\hline $\mathrm{C} 3 \mathrm{~B}-\mathrm{C} 1 \mathrm{~B}-\mathrm{C} 17 \mathrm{~B}-\mathrm{C} 21 \mathrm{~B}$ & $178.69(13)$ \\
\hline $\mathrm{C} 22 \mathrm{~B}-\mathrm{C} 17 \mathrm{~B}-\mathrm{C} 18 \mathrm{~B}-\mathrm{C} 19 \mathrm{C}$ & $-127.4(4)$ \\
\hline $\mathrm{C} 1 \mathrm{~B}-\mathrm{C} 17 \mathrm{~B}-\mathrm{C} 18 \mathrm{~B}-\mathrm{C} 19 \mathrm{C}$ & $112.1(4)$ \\
\hline $\mathrm{C} 21 \mathrm{~B}-\mathrm{C} 17 \mathrm{~B}-\mathrm{C} 18 \mathrm{~B}-\mathrm{C} 19 \mathrm{C}$ & $-6.0(4)$ \\
\hline $\mathrm{C} 22 \mathrm{~B}-\mathrm{C} 17 \mathrm{~B}-\mathrm{C} 18 \mathrm{~B}-\mathrm{C} 19 \mathrm{~B}$ & $-158.39(17)$ \\
\hline $\mathrm{C} 1 \mathrm{~B}-\mathrm{C} 17 \mathrm{~B}-\mathrm{C} 18 \mathrm{~B}-\mathrm{C} 19 \mathrm{~B}$ & $81.06(19)$ \\
\hline $\mathrm{C} 21 \mathrm{~B}-\mathrm{C} 17 \mathrm{~B}-\mathrm{C} 18 \mathrm{~B}-\mathrm{C} 19 \mathrm{~B}$ & $-37.00(19)$ \\
\hline $\mathrm{C} 19 \mathrm{C}-\mathrm{C} 18 \mathrm{~B}-\mathrm{C} 19 \mathrm{~B}-\mathrm{C} 20 \mathrm{~B}$ & $-79.9(7)$ \\
\hline $\mathrm{C} 17 \mathrm{~B}-\mathrm{C} 18 \mathrm{~B}-\mathrm{C} 19 \mathrm{~B}-\mathrm{C} 20 \mathrm{~B}$ & $24.8(2)$ \\
\hline $\mathrm{C} 19 \mathrm{~B}-\mathrm{C} 18 \mathrm{~B}-\mathrm{C} 19 \mathrm{C}-\mathrm{C} 20 \mathrm{~B}$ & $61.7(7)$ \\
\hline $\mathrm{C} 17 \mathrm{~B}-\mathrm{C} 18 \mathrm{~B}-\mathrm{C} 19 \mathrm{C}-\mathrm{C} 20 \mathrm{~B}$ & $-23.0(5)$ \\
\hline $\mathrm{C} 18 \mathrm{~B}-\mathrm{C} 19 \mathrm{~B}-\mathrm{C} 20 \mathrm{~B}-\mathrm{C} 21 \mathrm{~B}$ & $-2.1(3)$ \\
\hline $\mathrm{C} 18 \mathrm{~B}-\mathrm{C} 19 \mathrm{~B}-\mathrm{C} 20 \mathrm{~B}-\mathrm{C} 19 \mathrm{C}$ & $71.2(6)$ \\
\hline $\mathrm{C} 18 \mathrm{~B}-\mathrm{C} 19 \mathrm{C}-\mathrm{C} 20 \mathrm{~B}-\mathrm{C} 19 \mathrm{~B}$ & $-69.4(6)$ \\
\hline $\mathrm{C} 18 \mathrm{~B}-\mathrm{C} 19 \mathrm{C}-\mathrm{C} 20 \mathrm{~B}-\mathrm{C} 21 \mathrm{~B}$ & $43.0(4)$ \\
\hline $\mathrm{C} 19 \mathrm{~B}-\mathrm{C} 20 \mathrm{~B}-\mathrm{C} 21 \mathrm{~B}-\mathrm{C} 17 \mathrm{~B}$ & $-21.4(2)$ \\
\hline $\mathrm{C} 19 \mathrm{C}-\mathrm{C} 20 \mathrm{~B}-\mathrm{C} 21 \mathrm{~B}-\mathrm{C} 17 \mathrm{~B}$ & $-48.7(3)$ \\
\hline $\mathrm{C} 22 \mathrm{~B}-\mathrm{C} 17 \mathrm{~B}-\mathrm{C} 21 \mathrm{~B}-\mathrm{C} 20 \mathrm{~B}$ & $159.15(14)$ \\
\hline $\mathrm{C} 1 \mathrm{~B}-\mathrm{C} 17 \mathrm{~B}-\mathrm{C} 21 \mathrm{~B}-\mathrm{C} 20 \mathrm{~B}$ & $-82.06(16)$ \\
\hline $\mathrm{C} 18 \mathrm{~B}-\mathrm{C} 17 \mathrm{~B}-\mathrm{C} 21 \mathrm{~B}-\mathrm{C} 20 \mathrm{~B}$ & $36.00(16)$ \\
\hline $\mathrm{C} 23 \mathrm{~B}-\mathrm{N} 3 \mathrm{~B}-\mathrm{C} 22 \mathrm{~B}-\mathrm{O} 5 \mathrm{~B}$ & $-1.9(2)$ \\
\hline $\mathrm{C} 23 \mathrm{~B}-\mathrm{N} 3 \mathrm{~B}-\mathrm{C} 22 \mathrm{~B}-\mathrm{C} 17 \mathrm{~B}$ & $175.88(14)$ \\
\hline $\mathrm{C} 1 \mathrm{~B}-\mathrm{C} 17 \mathrm{~B}-\mathrm{C} 22 \mathrm{~B}-\mathrm{O} 5 \mathrm{~B}$ & $-5.3(2)$ \\
\hline $\mathrm{C} 18 \mathrm{~B}-\mathrm{C} 17 \mathrm{~B}-\mathrm{C} 22 \mathrm{~B}-\mathrm{O} 5 \mathrm{~B}$ & $-128.31(16)$ \\
\hline $\mathrm{C} 21 \mathrm{~B}-\mathrm{C} 17 \mathrm{~B}-\mathrm{C} 22 \mathrm{~B}-\mathrm{O} 5 \mathrm{~B}$ & $116.19(16)$ \\
\hline $\mathrm{C} 1 \mathrm{~B}-\mathrm{C} 17 \mathrm{~B}-\mathrm{C} 22 \mathrm{~B}-\mathrm{N} 3 \mathrm{~B}$ & $176.90(14)$ \\
\hline $\mathrm{C} 18 \mathrm{~B}-\mathrm{C} 17 \mathrm{~B}-\mathrm{C} 22 \mathrm{~B}-\mathrm{N} 3 \mathrm{~B}$ & $53.86(19)$ \\
\hline $\mathrm{C} 21 \mathrm{~B}-\mathrm{C} 17 \mathrm{~B}-\mathrm{C} 22 \mathrm{~B}-\mathrm{N} 3 \mathrm{~B}$ & $-61.63(19)$ \\
\hline $\mathrm{C} 22 \mathrm{~B}-\mathrm{N} 3 \mathrm{~B}-\mathrm{C} 23 \mathrm{~B}-\mathrm{C} 24 \mathrm{~B}$ & $-135.46(15)$ \\
\hline $\mathrm{C} 22 \mathrm{~B}-\mathrm{N} 3 \mathrm{~B}-\mathrm{C} 23 \mathrm{~B}-\mathrm{C} 28 \mathrm{~B}$ & $101.30(17)$ \\
\hline $\mathrm{N} 3 \mathrm{~B}-\mathrm{C} 23 \mathrm{~B}-\mathrm{C} 24 \mathrm{~B}-\mathrm{C} 25 \mathrm{~B}$ & $174.80(13)$ \\
\hline $\mathrm{C} 28 \mathrm{~B}-\mathrm{C} 23 \mathrm{~B}-\mathrm{C} 24 \mathrm{~B}-\mathrm{C} 25 \mathrm{~B}$ & $-60.90(17)$ \\
\hline $\mathrm{C} 23 \mathrm{~B}-\mathrm{C} 24 \mathrm{~B}-\mathrm{C} 25 \mathrm{~B}-\mathrm{C} 26 \mathrm{~B}$ & $59.43(17)$ \\
\hline $\mathrm{C} 24 \mathrm{~B}-\mathrm{C} 25 \mathrm{~B}-\mathrm{C} 26 \mathrm{~B}-\mathrm{C} 27 \mathrm{~B}$ & $-55.29(19)$ \\
\hline $\mathrm{C} 25 \mathrm{~B}-\mathrm{C} 26 \mathrm{~B}-\mathrm{C} 27 \mathrm{~B}-\mathrm{C} 28 \mathrm{~B}$ & $52.8(2)$ \\
\hline $\mathrm{N} 3 \mathrm{~B}-\mathrm{C} 23 \mathrm{~B}-\mathrm{C} 28 \mathrm{~B}-\mathrm{C} 27 \mathrm{~B}$ & $-178.54(13)$ \\
\hline $\mathrm{C} 24 \mathrm{~B}-\mathrm{C} 23 \mathrm{~B}-\mathrm{C} 28 \mathrm{~B}-\mathrm{C} 27 \mathrm{~B}$ & $57.82(18)$ \\
\hline $\mathrm{C} 26 \mathrm{~B}-\mathrm{C} 27 \mathrm{~B}-\mathrm{C} 28 \mathrm{~B}-\mathrm{C} 23 \mathrm{~B}$ & $-53.88(19)$ \\
\hline
\end{tabular}




\section{supporting information}

Hydrogen-bond geometry $\left(\AA,{ }^{\circ}\right)$

\begin{tabular}{lllll}
\hline$D-\mathrm{H} \cdots A$ & $D-\mathrm{H}$ & $\mathrm{H} \cdots A$ & $D \cdots A$ & $D-\mathrm{H} \cdots A$ \\
\hline $\mathrm{N} 2 A-\mathrm{H} 2 A A \cdots \mathrm{O} 5 A$ & 0.88 & 2.09 & $2.9371(18)$ & 161 \\
$\mathrm{~N} 3 A-\mathrm{H} 3 A A \cdots \mathrm{O} 1 W$ & 0.88 & 2.00 & $2.8662(19)$ & 170 \\
$\mathrm{~N} 2 B-\mathrm{H} 2 B A \cdots \mathrm{O} 5 B$ & 0.88 & 2.10 & $2.9330(18)$ & 157 \\
$\mathrm{~N} 3 B-\mathrm{H} 3 B A \cdots \mathrm{O} 3 A$ & 0.88 & 2.07 & $2.9238(17)$ & 163 \\
$\mathrm{O} 1 W-\mathrm{H} 1 W A \cdots \mathrm{O} 4 B^{\mathrm{i}}$ & $0.85(3)$ & $1.90(3)$ & $2.7433(18)$ & $169(2)$ \\
$\mathrm{O} 1 W-\mathrm{H} 1 W B \cdots \mathrm{O} 4 A^{\mathrm{ii}}$ & $0.89(2)$ & $1.88(2)$ & $2.7569(18)$ & $170(2)$
\end{tabular}

Symmetry codes: (i) $-x+1,-y+2,-z+1$; (ii) $x+1, y, z$. 